\title{
7. Die Ära Willi Arens (1990-1998)
}

\subsection{Vom Boom zum Absturz}

Mit der deutschen Wiedervereinigung brach auch für die GTB eine neue Zeit an. Schon lange zuvor war der Führungswechsel geplant worden, der auf dem 16. Ordentlichen Gewerkschaftstag im November 1990 anstand. Willi Arens war bereits seit 1984 Mitglied des Geschäftsführenden Hauptvorstandes (GHV) der Textilgewerkschaft. Bei seiner damaligen Wahl hatte sich die sogenannte »Familie«, der bereits in den 1950er Jahren ins Leben gerufene und die GTB seit über zwanzig Jahren informell führende Kreis der »Modernisierer«, auf Arens als designierten Vorsitzenden verständigt.

Willi Arens war gelernter Weber und trat 1964 eine Stelle als Gewerkschaftssekretär in Nordhorn an. Von 1970 bis 1986 gehörte er dem Niedersächsischen Landtag an und war zuletzt wirtschaftspolitischer Sprecher der SPD-Fraktion. Für die GTB-Vorstandsposition gab Arens seine parteipolitische Karriere auf. Mit dieser Personalie wollte Berthold Keller den gemäßigten politischen Kurs langfristig festigen. Dennoch bedeutete Arens' Wahl kein einfaches »Weiter so«, denn die Nachkriegsgeneration, die noch persönlich an den Auseinandersetzungen der 1950er Jahre um den politischen Kurs der GTB beteiligt war, verlor an Bedeutung. Wolfgang Stender, den der Beirat 1989 für Hermann Schumacher nachgewählt hatte, war das letzte GHV-Mitglied, das persönlich an der politischen Wende zu Beginn der 1960er Jahre beteiligt war.

Auf dem Gewerkschaftstag wählten die noch ausschließlich in Westdeutschland nominierten Delegierten die neue Führung für die nächsten vier gesamtdeutschen Jahre, ohne die inzwischen beschlossene Auflösung der DDR-Gewerkschaft IG TeBeLe zu berücksichtigen. Nur 162 der 209 Delegierten, also 77,5 Prozent wählten Arens zum Vorsitzenden. Sein Stellvertreter, Hermann Paschen, erhielt 180 Stimmen, für den Hauptkassierer Serv Hennes stimmten 164 Delegierte. Von den übrigen GHV-Mitgliedern bekamen der vom Beirat nachgewählte Manfred Schallmeyer 190, Waltraud Hessedenz 153 und der ebenfalls vom Beirat nachgewählte Tarifchef Wolfgang Stender 126 Stimmen.

Das neue Führungsteam sah sich bisher nicht gekannten Herausforderungen gegenüber. Seit Gründung der Bundesrepublik befanden sich die Textil- und 
die Bekleidungsindustrie in ständigem Auf und Ab, wobei die »Abs « in der Regel stärker ausfielen. Diese Volatilität verschärfte sich im vereinigten Deutschland. Die ostdeutsche Textil- und Bekleidungsindustrie war sehr beschäftigungsstark und hatte fast so viele Beschäftigte wie ihre Schwesterbranchen in Westdeutschland. Zunächst profitierte jedoch nur der Westen vom Einigungsboom; 1990/1991 schnellten die Umsätze um 10 Prozent in die Höhe. ${ }^{1}$ Die so realisierten tarifpolitischen Höhenflüge mündeten jedoch abrupt in eine existenzielle Krise des Flächentarifvertrags. Anders sah die Situation in Ostdeutschland aus, wo die GTB trotz des plötzlichen Zusammenbruchs des Industriezweigs eine dort bisher unbekannte, von staatlichen Einflüssen unabhängige Tarifpolitik aufbauen musste. Die in Ost und West bestehende Erwartungshaltung von Mitgliedern, dass sich der unter der Regierung Kohl forcierte Sozialabbau durch Tarifpolitik kompensieren ließe, verkomplizierte die Lage weiter.

\subsubsection{Ostdeutschland: Der schnelle Tod einer Branche}

Ein Großteil der ostdeutschen Textil- und Bekleidungsproduktion wurde zu DDRZeiten exportiert, auch in die Bundesrepublik. Hierbei spielte der offizielle Kurs der DDR-Mark zur westdeutschen D-Mark keine Rolle; beim Export erzielte die DDR für eine Ostmark 0,23 DM, was einem Kurs von 4,4 zu 1 entsprach. ${ }^{2}$ Aufgrund der bisherigen Lieferungen in das "Nichtsozialistische Wirtschaftsgebiet« gab es durchaus optimistische Prognosen zur Branchenzukunft. Innerhalb der GTB-Führung herrschte die Einschätzung vor, mittelfristig könnten zumindest 100.000 der 300.000 ostdeutschen Arbeitsplätze erhalten werden, was sich jedoch rasch als Fehlprognose herausstellte.

Die Währungsunion brachte der DDR-Mark den 1:1-Umrechnungskurs und führte zur »Aufwertung « der ostdeutschen Währung, wodurch sich der D-MarkKaufpreis für ostdeutsche Waren mehr als vervierfachte. Material- oder Lohnkosten der ostdeutschen Unternehmen im gleichen Umfang zu senken war unmöglich. Hinzu kam, dass die Absatzmärkte in den realsozialistischen Staaten wegbrachen, weil die Abnehmerländer nicht in der Lage waren, die erhöhten Preise in frei konvertierbarer Währung zu bezahlen. Darüber hinaus waren Abläufe und Organisation in den riesigen textilen Kombinaten und Volkseigenen Betrieben (VEB) unproduktiv und schwerfällig. Die Bundesregierung und die zur Privatisierung der VEB eingesetzte Treuhandanstalt blieben passiv.

Am 18. März 1991 gingen mehr als 40.000 Textiler:innen in Chemnitz, Cottbus und Löbau auf die Straße und forderten unter dem Motto »Sanierung statt Liquidierung« lautstark eine radikale Wende in der Textilpolitik für die neuen Bundes-

1 Statistisches Bundesamt (Destatis) (2008): Fachserie 4, Reihe 4.1.1.

2 Sinn/Sinn (1992): Kaltstart, S. 72. 
länder. ${ }^{3}$ Trotz der unzähligen Berater und Experten gelang es der Treuhand nicht, die Rettung größerer Einheiten der Textil- und Bekleidungsindustrie zu organisieren; nur wenige Betriebe wurden von westdeutschen Unternehmen übernommen. Bereits Ende 1991 waren zwei Drittel der Branchenarbeitsplätze vernichtet und es gab nur noch knapp 97.000 textile Beschäftigte in Ostdeutschland. ${ }^{4}$

Angesichts dieser dramatischen Entwicklung verstärkte die GTB Ende 1991 den Druck auf Treuhand und Landesregierungen. Sie erreichte die Zusage, die noch existierenden Betriebe und deren Erhaltungswürdigkeit gezielt nach struktur- und regionalpolitischen Kriterien zu prüfen. Die Betriebe sollten befristete Lohnkostenzuschüsse erhalten, um global wettbewerbsfähig zu werden. 200.000 Arbeitsplätze hatte das »Nichthandeln« der Treuhand bisher gekostet; bei weiterhin rapiden Arbeitsplatzverlusten hoffte man jetzt, noch 40.000 Arbeitsplätze erhalten zu können. ${ }^{5}$ Aber auch diese Hoffnung sollte trügen - binnen zwei Jahren war dieser große Industriezweig der ostdeutschen Konsumgüterindustrie nahezu komplett verschwunden. Dies war ein beispielloser Vorgang in der neueren Wirtschaftsgeschichte.

1993 gab es nur noch rund 23.000 Beschäftigte in den fünf ostdeutschen Bundesländern, ${ }^{6}$ was sich zwangsläufig in der Mitgliederentwicklung der GTB niederschlug. Von über 200.000 Mitgliedern der IG TeBeLe im Jahr 1990 waren Ende 1991 noch gut 100.000 in der GTB organisiert, zwei Jahre später nur noch 20.000, 1995 waren es nur noch 10.000. Von zwanzig gewerkschaftlichen Betreuungsstellen blieben sieben hauptamtlich besetzte Verwaltungsstellen sowie zwei WestOst-Verwaltungsstellen übrig. ${ }^{8}$

Neben dem Mitgliederrückgang aufgrund von Arbeitsplatzvernichtung sank auch der Organisationsgrad in den verbliebenen Betrieben. Die von der GTB angestellten ehemaligen DDR-Gewerkschafter:innen waren hoch motiviert und in den Augen vieler Beschäftigter glaubwürdiger als die »Besserwessis«. Sie verstanden die Mentalität der ehemaligen DDR-Bürger:innen und spürten die Auswirkungen des Umbruchs im Familien- und Freundeskreis unmittelbarer als "personelle Westimporte«, verfügten aber oft nicht über ausreichende Kenntnisse und Erfahrungen im Umgang mit der kapitalistischen Wirtschaftsweise. Vor allem scheuten sie sich, den oft erforderlichen »milden Druck« auszuüben, um neue Mitglieder zu rekrutieren und ehemalige zurückzugewinnen.

3 textil-bekleidung, Ausgabe 4/1991, S. 3 und $14 \mathrm{f}$.

4 GTB: Geschäftsbericht 1990-1993 des Hauptvorstandes, S. 85.

5 textil-bekleidung, Ausgabe 1/1992, S. 3.

6 Statistisches Bundesamt (Destatis) (2008): Fachserie 4, Reihe 4.1.1.

7 CTB: Ceschäftsbericht 1990-1993 des Hauptvorstandes, S. 243; CTB: Ceschäftsbericht 1994-1997 des Hauptvorstandes, S. $237 \mathrm{ff}$.

8 GTB: Ceschäftsbericht 1999-1993 des Hauptvorstandes, S. $261 \mathrm{ff}$. 
Angesichts der katastrophalen wirtschaftlichen Entwicklung in der ehemaligen DDR blieben die tariflichen Erfolge bescheiden. Im Vordergrund standen Aktivitäten zum Erhalt der Arbeitsplätze, die aber für über 90 Prozent der Beschäftigten vergeblich waren. Im September 1990 hatte die GTB die Verhandlungsführung für das ostdeutsche Tarifgebiet übernommen. Ihr standen zunächst sechzehn Arbeitgeberverbände gegenüber, aber innerhalb der nächsten zwei Jahre setzte sich der vti (Verband der Nordostdeutschen Textilindustrie, später Verband der Nordostdeutschen Textil- und Bekleidungsindustrie) als alleinige Tarifvertragspartei des Arbeitgeberlagers durch. Die anderen Verbände gingen im vti auf, sofern sie nicht mangels Mitgliedern von der Bildfläche verschwanden.

Die GTB konnte die Struktur der westdeutschen Tarifverträge 1991 grundsätzlich auf die neuen Bundesländer übertragen und die Einkommen auf 60 Prozent des Westniveaus erhöhen. Die Arbeitszeit wurde von 43,75 Stunden pro Woche auf 40 Stunden abgesenkt, die Urlaubsdauer auf sechs Wochen im Jahr erhöht. ${ }^{9}$ Jahressonderzahlungen und Urlaubsgeld blieben unterhalb des Niveaus der alten Bundesländer. In den Folgejahren ging es in kleineren Stufen weiter. Zwar gelang mit jedem Tarifabschluss eine Annäherung an die Standards in den westlichen Bundesländern, jedoch blieb die Tarifbindung im Osten deutlich geringer als in Westdeutschland. Dies war für die Angleichung der Arbeitsbedingungen wenig hilfreich. Im GTB-Geschäftsbericht wurden auch »vielfach vorgenommene betriebliche Zugeständnisse « beklagt. ${ }^{10}$

Als die Tarifverhandlungen für die ostdeutsche Textilindustrie 1995 stockten, rief die GTB dort erstmals zu Warnstreiks auf. Zur Überraschung der Arbeitgeber legten die Belegschaften in sieben Betrieben zeitweise die Arbeit nieder. ${ }^{11}$ Die GTB hatte sich trotz des großen Aderlasses auch in der Textilindustrie Sachsens und Thüringens als aktionsfähig bewiesen. Während die Angleichung ans Westniveau in der ostdeutschen Textilindustrie in kleinen Schritten weiterging, brach die GTB die Tarifgespräche für die ostdeutsche Bekleidungsindustrie $1995 \mathrm{ab}$. Grund war die inzwischen sehr geringe Anzahl tarifgebundener Betriebe. ${ }^{12}$ Bis heute existiert der Flächentarifvertrag für die ostdeutsche Bekleidungsindustrie nur noch in der Nachwirkung, ${ }^{13}$ aber auch die Tarifbindung der Textilindustrie im Osten Deutschlands blieb gering.

9 GTB: Geschäftsbericht 1990-1993 des Hauptvorstandes, S. 284.

10 GTB: Geschäftsbericht 1994-1997 des Hauptvorstandes, S. 262.

11 GTB: Geschäftsbericht 1994-1997 des Hauptvorstandes, S. 285.

12 GTB: Geschäftsbericht 1994-1997 des Hauptvorstandes, S. 263.

13 Nach Ablauf eines Tarifvertrags gelten seine Rechtsnormen weiter, bis sie durch eine andere Abmachung ersetzt werden ( $\$ 4$ Absatz 5 Tarifvertragsgesetz). Deshalb gelten die Regeln eines 
Anfang des neuen Jahrtausends gelang es der IG Metall, mit dem ersten gemeinsamen Entgelttarifvertrag für Arbeiter:innen und Angestellte in dieser Branche gesamtdeutsche Tarifgeschichte zu schreiben. In der Textilindustrie wurde 2019 noch vor der Metall- und Elektroindustrie die Anpassung der regelmäßigen wöchentlichen Arbeitszeit vereinbart. Bis 2027 wird die Arbeitszeit der Beschäftigten in den ostdeutschen Textilbetrieben schrittweise von 40 auf die im Westen gültigen 37 Wochenstunden angeglichen. Die Beschäftigtenzahl der ostdeutschen Textilindustrie hat sich seit 1993 auf einem niedrigen Niveau stabilisiert, nennenswerte Bekleidungsbetriebe gibt es dort nicht mehr.

\subsubsection{West: Die Welle trägt noch}

Nach dem bereits 1990 einsetzenden Wiedervereinigungsboom erhöhte sich die Wirtschaftsleistung in den alten Bundesländern 1991 um über 5 Prozent; ${ }^{14}$ die Preissteigerungsrate stieg auf 3,7 Prozent. ${ }^{15}$ Die GTB sah angesichts des zuletzt niedrigen Abschlusses und des Wirtschaftsaufschwungs einen großen Nachholbedarf und forderte 1991 eine Entgeltsteigerung von 10 Prozent sowie weitere Schritte in Richtung 35-Stunden-Woche bei vollem Lohnausgleich. In der westdeutschen Textil- und Bekleidungsindustrie war die wöchentliche Arbeitszeit in den letzten drei Jahren bereits von 40 auf 38,5 Stunden verkürzt worden. Die Arbeitgeber wandten sich insbesondere gegen die Arbeitszeitverkürzung. Erneut machte die Gewerkschaft Druck mit massiven Warnstreiks. Im Ergebnis erzielte sie für die Textilindustrie Einkommensverbesserungen von 6,7 Prozent und die weitere Verkürzung der Wochenarbeitszeit bis Ende 1993 auf 37 Stunden.

In der Bekleidungsindustrie verweigerten die Arbeitgeber die Übernahme dieses Tarifabschlusses. Nach der zweiten Warnstreikwelle, an der sich rund die Hälfte der Mitglieder beteiligte, stimmten Anfang Juni 1991 auch die Bekleidungshersteller der Lohn- und Gehaltserhöhung von 6,7 Prozent zu. Die Arbeitszeitverkürzung wurde gegenüber der Textilindustrie etwas abgebremst und mit drei Halbstunden-Schritten bis 1994 gestreckt. Für beide westdeutsche Tarifgebiete musste die GTB jedoch Zugeständnisse bei der Arbeitszeitflexibilisierung machen: Der Ausgleichszeitraum, in dem die regelmäßige tarifliche Wochenarbeits-

Tarifvertrags - auch wenn er gekündigt wurde - für die bereits vor der Kündigung Beschäftigten »ewig«weiter.

14 Statistisches Bundesamt (Destatis) (2018): Volkswirtschaftliche Gesamtrechnungen, Inlandsproduktberechnungen ab 1970, S. 14

15 Statistisches Bundesamt (Destatis) (2021): Preise. Verbraucherpreisindizes. Lange Reihen ab 1948, JD-Index. 
zeit durchschnittlich erreicht werden musste, wurde von 18 auf 26 Wochen verlängert. ${ }^{16}$

In der Einkommenspolitik hatte die GTB das mittelfristige Ziel der Zusammenfassung der Lohn- und Gehaltstarifverträge zu einem gemeinsamen textilen Entgeltrahmentarifvertrag (tera) postuliert. Damit sollten Unterschiede in der Bewertung der Qualifikation von Arbeiter:innen und Angestellten ausgeglichen und gewerbliche Tätigkeiten aufgewertet werden. Als erster Schritt wurden Reformen der Lohntarifverträge angestrebt. Oberbegriffe und Richtbeispiele sollten die bisherigen detaillierten Tätigkeitsbeschreibungen ersetzen, die teilweise durch die technische Entwicklung und arbeitsorganisatorische Veränderungen überholt waren.

Auch der Jahresbeginn 1992 war noch vom Wirtschaftsboom der beiden Vorjahre geprägt. Der Hauptvorstand strebte ein Forderungsvolumen von 9 Prozent an; auf Druck der regionalen Tarifkommissionen wurde schließlich eine Forderung von 9,5 Prozent beschlossen. Zusätzlich sollte ein Tarifvertrag zur Aus-, Fort- und Weiterbildung die in den Branchen herrschende Stufenausbildung im Sinne der Auszubildenden verbessern und das Recht auf Weiterbildung für alle Beschäftigten verankern. Außerdem sollten Schritte in Richtung des angestrebten Entgeltrahmentarifvertrags tera vereinbart werden.

Auch 1992 unterstützten 70.000 Beschäftigte die Forderungen durch Warnstreiks. Doch erst nach Urabstimmungen und Streikbeginn in Baden-Württemberg und Nordrhein wurde schließlich in der baden-württembergischen Textilindustrie der Durchbruch erzielt. "Das Tor zur Zukunft [ist] geöffnet", kommentierte Wolfgang Stender das im Juni 1992 erzielte Tarifergebnis. ${ }^{17}$ In der Tat war ein deutlicher Schritt in Richtung tera gelungen: Für Arbeiter:innen wurden anstatt der bisher geltenden Stundenlöhne gleichbleibende Monatslöhne eingeführt, für qualifizierte gewerbliche Tätigkeiten gelang mit drei zusätzlichen Lohngruppen ein Angleichungsschritt an die Gehälter und für Beschäftigte im Leistungslohn wurde eine Mindestabsicherung von 115 Prozent des Prämien- oder Akkordrichtsatzes festgelegt.

Angesichts dieser fühlbaren strukturellen Verbesserungen bestanden die Arbeitgeber auf einer zweijährigen Laufzeit. Die Einkommen erhöhten sich 1992 und 1993 jeweils um 4,1 Prozent; hinzu kam der Lohnausgleich in Höhe von 2,8 Prozent für die bereits im Vorjahr vereinbarte Arbeitszeitverkürzung von einer Stunde. Anders als in Baden-Württemberg legten die übrigen regionalen textilen Tarifkommissionen keinen Schwerpunkt auf die tera-Forderung. Deshalb fielen die Lohnerhöhungen mit 4,3 Prozent (1992) und 4,0 Prozent (1993) ins-

16 CTB: Ceschäftsbericht 1990-1993 des Hauptvorstandes, S. $285 \mathrm{ff}$.

17 Stender (1992): Das Tor zur Zukunft geöffnet, S. 6. 
gesamt geringfügig höher aus als in Baden-Württemberg, ebenfalls zuzüglich Lohnausgleich für die Arbeitszeitverkürzung.

In der Bekleidungsindustrie wurden die Löhne und Gehälter im Jahr 1992 um 4,4 Prozent erhöht und die höchste Lohngruppe für die Tätigkeit der »Musternäherin« durchgesetzt. Die Stundenlöhne wurden zusätzlich um 1,4 Prozent erhöht, um die Verdienstminderung wegen der dreißigminütigen Arbeitszeitverkürzung auszugleichen. Abweichend von der Textilindustrie wurde der Bekleidungstarifvertrag nur für ein Jahr abgeschlossen.

\subsubsection{Am Rande des Abgrunds: Die Jahre 1993 und 1994}

Im Laufe des Jahres 1992 verlief die wirtschaftliche Entwicklung in Deutschland anders als erhofft. In der zweiten Jahreshälfte brach die Konjunktur ein, 1993 schrumpfte die Wirtschaftsleistung sogar - Deutschland war in eine Rezession abgerutscht. Der westdeutsche Textilumsatz sank 1992 im Vergleich zum Vorjahr um 4,5 Prozent, in der Bekleidungsindustrie um 6,8 Prozent. Im Folgejahr kamen weitere Rückgänge im zweistelligen Bereich hinzu. Die GTB forderte politische Unterstützung für die Branchen. Arbeitgeberverbände und Gewerkschaft schilderten im September 1992 die dramatische Lage bei einem Spitzengespräch im Kanzleramt.

Der GTB-Vorsitzende Willi Arens rechnete vor, dass sich der Importüberschuss innerhalb von nur zwei Jahren von 11,7 Milliarden DM in 1989 auf 24,5 Milliarden DM in 1991 mehr als verdoppelt hatte. Er warnte vor den katastrophalen Folgen im Falle einer weiteren Marktöffnung und forderte die Regierung auf, sich gegen das Auslaufen des Welttextilabkommens $1992 \mathrm{zu}$ wenden und stattdessen Verbesserungen einzufordern. Künftig sollten nur die Länder einen Markzugang erhalten, die die Kernarbeitsnormen der ILO einhielten und die europäischen Umweltvorschriften nicht unterliefen. Außerdem müsse im Gegenzug der Markzugang deutscher Hersteller zu den Lieferländern liberalisiert werden. Die Arbeitgeberseite forderte darüber hinaus ein wirksames Antidumping- und Antisubventionsrecht sowie einen besseren Schutz gegen den sogenannten Musterklau. Für die neuen Bundesländer forderte die GTB die staatliche Finanzierung eines »Übergangsregimes«, bis die Branchen sich marktwirtschaftlich behaupten könnten. So hoffte man, den Kern der Branchen zu erhalten. ${ }^{18}$

Die Regierung beschränkte sich jedoch auf verbale Streicheleinheiten und leitete keine entsprechenden Maßnahmen ein. Ab Ende 1992 verstärkte die GTB den öffentlichen Druck durch regionale Protestkundgebungen, die teilweise gemeinsam mit den Arbeitgeberverbänden organisiert wurden. »Bonn, wir kommen«, titelte die Mitgliederzeitschrift textil-bekleidung im Dezember 1992:

18 textil-bekleidung, Ausgabe 9/1992, S. 3, 6 f. und 10 (»]etzt muss der Kanzler ran«). 
»Die Zeit der vornehmen Zurückhaltung ist vorbei. Unternehmer der deutschen Textil- und Bekleidungsindustrie und die Cewerkschaft Textil-Bekleidung werden bei der Wahrnehmung der Interessen einen Zahn zulegen. ${ }^{19}$

Im März 1993 versammelten sich schließlich 2.200 Unternehmer:innen, leitende Angestellte, Betriebsräte und Gewerkschafter:innen zur »Deutschen Textil- und Bekleidungs-Konferenz« in Bonn. Bundeswirtschaftsminister Günter Rexrodt und EU-Kommissar Peter Schmidhuber verteidigten dabei grundsätzlich den liberalen Welthandel, sagten aber mehr Aktivitäten gegen Markenpiraterie, Schutz vor Dumping und unlauteren Staatshilfen zu.

Am 18. Juni 1993 debattierte der Deutsche Bundestag über die Lage der Textilund der Bekleidungsindustrie. Die GTB begleitete die Debatte mit einer Demonstration in Bonn. In allen Verwaltungsstellen hatten die Gewerkschafter:innen Stoffbahnen mit Forderungen beschrieben, die vor Ort um »fünf vor zwölf« symbolträchtig zu einem 8 Kilometer langen Transparent zusammengenäht wurden. Unter dem Motto »Wir machen Stoff für unsere Arbeitsplätze« verbanden 3.000 Demonstrierende Wirtschaftsministerium und Kanzleramt mit dem »längsten Transparent der Welt« und blockierten die Bonner Innenstadt. Die zeitgleich laufende Bundestagsdebatte verlief allerdings enttäuschend. Wirtschaftsminister Rexrodt blieb allgemein, forderte Steuersenkungen und flexiblere Arbeitszeiten. Immerhin sprach sich der Bundestag für weitere internationale Textilabkommen aus, forderte die Harmonisierung der Umweltauflagen in der EG sowie die Abwehr von Quotenüberschreitungen und Umgehungen beim internationalen Textilhandel. ${ }^{20}$

GTB und Textil-Arbeitgeber waren sich in der Beurteilung der Strukturschwierigkeiten weitgehend einig und vertraten gegenüber der Politik die Brancheninteressen "Arm in Arm«, aber tarifpolitisch zogen dunkle Wolken auf. Die Verbände der Textilindustrie, die noch Mitte 1992 eine zweijährige Laufzeit der Tarifverträge durchgesetzt hatten, forderten nun eine Revision des Tarifabschlusses. Diesem Anliegen verweigerte sich die GTB nicht grundsätzlich, erwartete jedoch eine Vereinbarung, zu der beide Tarifparteien ihren Beitrag leisten. Seit Jahrzehnten hatte die GTB das Anliegen, die Fort- und Weiterbildung tarifpolitisch zu regeln; 1963 hatte sie dies im kleinen Tarifgebiet der Miederindustrie bereits erreicht (siehe Kapitel 4.1.2). Die Gewerkschaft stellte in Aussicht, die anstehende Arbeitszeitverkürzung von einer Stunde pro Woche um zwei Jahre zu verschieben, was die Betriebe um insgesamt 280 Millionen DM entlasten würde.

Im Gegenzug sollten die Arbeitgeber den Kapitalstock für eine Fort- und Weiterbildungs-Stiftung in Höhe von 18 bis 20 Millionen DM bereitstellen. Aus den

19 textil-bekleidung, Ausgabe 12/1992, S. 10.

20 textil-bekleidung, Ausgabe 7/8/1992, S. 3, 6f. und $10 \mathrm{f}$. 
Zinsen könnten Weiterbildungsmaßnahmen für die Beschäftigten finanziert werden. Gewerkschaftsmitglieder sollten durch Übernahme eines Teils der Lehrgangskosten oder durch Büchergelder »in geringem Umfange« gegenüber der Stiftung bessergestellt werden. ${ }^{21}$ Gesamttextil lehnte jedoch selbst eine geringe Besserstellung für Gewerkschaftsmitglieder kategorisch ab und die Arbeitgeber wollten nur maximal fünf Jahre lang je 500.000 DM in die Stiftung einbringen. Auch den Alternativvorschlag der GTB lehnte der Arbeitgeberverband ab: Statt der Einrichtung der Stiftung sollte die anstehende Arbeitszeit-Verkürzungsstufe auf 37 Wochenstunden verschoben werden; gleichzeitig sollten die Arbeitgeber einem Stufenplan zur weiteren Verkürzung der Arbeitszeit auf 35 Stunden pro Woche zustimmen.

Als die regionalen Arbeitgeberverbände den vermeintlichen Unwillen der GTB, zu einer gemeinsamen Lösung zu kommen, öffentlich kritisierten, reagierte die GTB mit einem offenen Brief, in dem Wolfgang Stender vom GHV schrieb:

»Die Unverhältnismäßigkeit Ihrer Vorschläge und die offenkundige Ceringschätzung unserer Überlegungen, die ja auch von der Akzeptanz der Mitglieder [...] abhängen und wegen der politischen Brisanz von besonderer Bedeutung sind, lassen die angestrebte Übereinkunft mit Ihnen nicht zu. $\aleph^{22}$

Nachdem eine Einigung mit der GTB nicht möglich schien, setzten viele Arbeitgeber die Beschäftigten und Betriebsräte unter Druck. Insbesondere wurde argumentiert, dass die Ende 1993 fällige Jahressonderzahlung, das dreizehnte Monatseinkommen zu Weihnachten, mit der vorhandenen Liquidität nicht zu stemmen sei. Wenn die Beschäftigten auf der Auszahlung bestünden, sei die Insolvenz nicht abzuwenden. Auch die Arbeitszeit war ein häufiges Streitthema. Vielfach forderten Arbeitgeber die betriebliche Rückkehr zur 40-Stunden-Woche, allerdings sollte nur die tarifliche Arbeitszeit von 37 Stunden bezahlt werden. Dies entspricht einem Lohnverzicht von 8 Prozent, der den Betrieb durch die schwierigen Zeiten bringen sollte. Jede dieser von den Arbeitgebern eingeforderten »Rettungsmaßnahmen« hätte die Gesamtkosten jedoch um höchstens 1,5 Prozent gesenkt, da der Lohn- und Gehaltskostenanteil am Umsatz bei rund 21 Prozent lag. ${ }^{23}$

Dennoch beugten sich Betriebsräte in nicht wenigen Fällen dem Druck und trafen entsprechende Vereinbarungen. Rechtlich waren diese Abmachungen illegal, legt doch das Tarifvertragsgesetz die Unabdingbarkeit von tarifvertraglichen

21 GTB: Geschäftsbericht 1990-1993 des Hauptvorstandes, S. 280.

22 Zit. nach: textil-bekleidung, Ausgabe 4/1993, S. 19.

23 Der Lohn- und Gehaltskostenanteil vom Umsatz lag 1992 in der Textilindustrie bei 20,67 Prozent; GTB: Geschäftsbericht 1990-1993 des Hauptvorstandes, S. 100. 
Normen fest. ${ }^{24}$ In den Fällen, bei denen Beschäftigten individuelle Verzichtserklärungen abgepresst wurden, lag die rechtliche Situation nicht anders. Unabhängig davon, ob die Kürzungen durch kollektive oder individuelle Abmachungen fixiert waren, hätte jedes GTB-Mitglied die Einhaltung des Tarifvertrags einklagen können, was aber nur in Einzelfällen passierte - in den Betrieben ging die Angst um. Im Zeitraum 1993/1994 war dieser Bruch von Tarifverträgen und Gesetzen keine Ausnahme; die GTB-Tarifabteilung schätzte, dass ungefähr die Hälfte der Beschäftigten Tarifleistungen in der einen oder anderen Form nicht erhielten.

Die Situation in der Bekleidungsindustrie unterschied sich von der in der Textilindustrie dahingehend, dass die Arbeitszeitverkürzungsstufen durch den Tarifabschluss 1991 über mehrere Jahre festgelegt, die Abkommen über Löhne und Gehälter 1992 jedoch nur auf zwölf Monate geschlossen waren. Während für die Textilindustrie Einkommenserhöhungen von 4 Prozent vereinbart waren, musste eine Lohnerhöhung für die Bekleidungsindustrie in der Krisensituation 1993 erst durchgesetzt werden. Bereits vor Beginn der Verhandlungen, in die die GTB mit einer Erhöhungsforderung von 6 Prozent ging, forderten die Arbeitgeber öffentlich, die 1991 vereinbarte Arbeitszeit-Verkürzungsstufe zu verschieben. Die vierte Verhandlung auf Bundesebene scheiterte am 26. Mai 1993, ebenso der erneute Einigungsversuch am 15. Juni. Die Arbeitgeber waren bei einer Inflationsrate von 3,6 Prozent ${ }^{25}$ nur zu Einkommensverbesserungen von 1,7 Prozent bereit. Nachdem Warnstreiks in 81 Betrieben die Arbeitgeber nicht an den Verhandlungstisch brachten, traten 5.500 GTB-Mitglieder in 36 Betrieben in einen auf zwei Tage befristeten Streik.

Nach dieser Machtdemonstration einigten sich der GTB-Vorsitzende Willi Arens und Fritz Goost, der Präsident der Bundesvereinigung der Arbeitgeber im BBI, in einem Spitzengespräch am 29. Juni 1993: Die Löhne und Gehälter stiegen um 2,1 Prozent und es blieb bei der Arbeitszeitverkürzung mit Lohnausgleich. Wichtig für die GTB war die Inkraftsetzung einer Strukturverbesserung. Die Gewerkschaft hatte sich in neunzehn Expertengesprächen mit der Arbeitgeberseite auf die Grundsätze eines neuen Lohnrahmentarifvertrages geeinigt. Damit war ein wichtiger Schritt zur diskriminierungsfreien Eingruppierung und in Richtung eines gemeinsamen Entgeltrahmentarifvertrags für Arbeiter:innen und Angestellte gelungen. Da dies jedoch eine erhebliche Kostenbelastung für die produzierenden Betriebe bedeutete, verständigte man sich auf ein stufenweises

24 Laut §4 Absatz 3 Tarifvertragsgesetz sind abweichende Abmachungen, die der Tarifvertrag selbst nicht gestattet, nur zulässig, wenn sie Regelungen zugunsten der Arbeitnehmer:innen enthalten. Ansonsten gelten die Normen für die tarifgebundenen Mitglieder »unmittelbar und zwingend«.

25 Statistisches Bundesamt (Destatis) (2021): Preise. Verbraucherpreisindizes. Lange Reihen ab 1948, JD-Index. 
Wirksamwerden. Als Erstes wurde die Höhergruppierung qualifizierter Tätigkeiten mit dem Tarifabschluss 1993 in Kraft gesetzt. ${ }^{26}$

Unabhängig von den gemeinsamen Initiativen zum Schutz der Arbeitsplätze vollzog sich die Tarifpolitik mit erstaunlicher Härte. In den Krisenjahren waren viele übertarifliche Bestandteile weggefallen und die Einkommen lagen deutlich unterhalb des Durchschnitts des Verarbeitenden Gewerbes. Vielfach war es unter dem Druck angedrohter Entlassungen zu illegalen Zugeständnissen gekommen. Dieselben Betriebsratsvorsitzenden, die ihren Widerstand gegen tarifwidrige Kürzungen innerhalb des Betriebs aufgegeben hatten und entsprechende Vereinbarungen trafen, drängten in den Tarifkommissionen auf eine harte Linie. So widersetzten sich die bezirklichen Tarifkommissionen, denen mehrere hundert Ehrenamtliche angehörten, Zugeständnissen auf der tarifvertraglichen Ebene. Dies war der Hintergrund, dass die GTB trotz des Beschäftigungsrückgangs von 8,7 Prozent und 13,2 Prozent in den Jahren 1992 und 1993 wieder einen Arbeitskampf organisierte, der zwar nicht in die Breite ging, aber mit der Beteiligung der Firmen Betty Barclay, Seidensticker, Gerry Weber, Boss und Hucke namhafte Player der Branche unter Druck setzte. ${ }^{27}$

Angesichts weiterer über 10-prozentiger Umsatzeinbrüche bei Textil und Bekleidung stand 1994 eine weitere Belastungsprobe an. Dies hatte sich bereits branchenübergreifend manifestiert: Die Arbeitgeber der Metall- und Elektroindustrie reagierten auf die IG Metall-Forderung nach 5,5 bis 6 Prozent Einkommenserhöhung mit der Gegenforderung einer Nullrunde. Gleichzeitig forderten sie den Wegfall des zusätzlichen Urlaubsgeldes und die Senkung der Mehrarbeitszuschläge. Mit der Kündigung der Tarifbestimmungen über Einmalzahlungen, also Urlaubs- und Weihnachtsgeld, verliehen sie ihrem Anliegen Nachdruck.

Erst nach massiven Warnstreiks, an denen sich fast 2 Millionen Metaller:innen beteiligten, und einer Urabstimmung gelang im Tarifgebiet Niedersachsen/ Bremen der Durchbruch. Nach fünf sogenannten Nullmonaten, in denen es keine Verbesserung gab, wurde eine 2-prozentige Erhöhung wirksam. Das Urlaubsgeld blieb unangetastet, allerdings wurde die Jahressonderzahlung 1994 einmalig um zehn Prozentpunkte gekürzt. Außerdem verlängerte sich der Verteilungsspielraum für die regelmäßige Wochenarbeitszeit auf 52 Wochen ohne jegliche einschränkende Regelung. Die Arbeitszeit konnte betrieblich von 36 auf bis zu 30 Stunden abgesenkt werden. Erfolgte im Gegenzug eine Beschäftigungssicherung, gab es keinen Lohnausgleich, ohne Beschäftigungssicherung einen Teillohnausgleich. ${ }^{28}$

26 CTB: Ceschäftsbericht 1990-1993 des Hauptvorstandes, S. $305 \mathrm{ff}$.

27 Vgl. Stender (1993): Hart errungene Kompromisse, S. 6f. und 10.

28 Quelle: WSI-Tarifarchiv, www.wsi.de/de/datenbank-tarifabschluesse-15320.htm (Abruf am 17.2.2021). 
Den ersten Tarifabschluss des Jahres verzeichnete zuvor jedoch die chemische Industrie. Für die Beschäftigten dieser Branche wurden die Einkommen nach drei Nullmonaten um 2 Prozent erhöht. Die tarifliche 37,5-Stunden-Woche konnte betrieblich um 2,5 Stunden gekürzt oder verlängert werden, womit die IG Chemie einen »Arbeitszeitkorridor« implementiert hatte.

Die GTB, bislang eine Vorreiterin bei der Gestaltung flexibler Arbeitszeiten, kam nun stark unter Druck. Für ihre früheren Zugeständnisse hatte sie Gegenleistungen wie Zuschläge in Form von Geld oder einen höheren Freizeitausgleich vereinbart. Nach den aktuellen Tarifergebnissen von Metall und Chemie sahen sich die Textilarbeitgeber deutlich schlechtergestellt als die Unternehmen der großen Industriebranchen. Die Mitglieder hingegen erwarteten von der GTB die Verteidigung der bestehenden Ausgewogenheit bei den Flexibilisierungsbestimmungen. Schon im Vorfeld der Tarifrunden 1994 hatte Willi Arens in der Januarausgabe der Mitgliederzeitschrift betont:

»Ziel der Tarifpolitik unserer Gewerkschaft wird es sein, ein Absinken des Lebensstandards der Beschäftigten zu verhindern. ${ }^{29}$

Allein die Verteidigung des Erreichten werde eine erhebliche Kraftanstrengung kosten, schrieb Arens an gleicher Stelle und sollte sich nicht täuschen. Bevor die GTB zu Anfang des Jahres über ihre Tarifforderungen diskutierte, hatten die Textil- und Bekleidungsarbeitgeber schon - der Marschroute von Gesamtmetall folgend - die Tarifverträge über zusätzliches Urlaubsgeld gekündigt. Sie drängten außerdem auf Wiedereinführung der 40-Stunden-Woche mit der Begründung, durch die zusätzlich geleisteten Stunden könnten die Beschäftigten den entstehenden Einkommensverlust wieder ausgleichen.

Eine besondere Strategie hatten sich die bayerischen Verbände ausgedacht: Der Verband der nordbayerischen Textilindustrie und der Verein der südbayerischen Textilindustrie hatten sich ebenso wie der Verband der bayerischen Bekleidungsindustrie Ende 1993 aufgelöst. Anstelle der alten Vereinigungen gründeten die Unternehmen den »Verband der bayerischen Textil- und Bekleidungsindustrie«, in dem sich sowohl die alten Vorstände als auch die Geschäftsführungen der aufgelösten Verbände wiederfanden. Die bayerischen Arbeitgeber gingen nun davon aus, dass wegen des Wegfalls der Tarifvertragsparteien auf Arbeitgeberseite alle bestehenden Tarifverträge gegenstandslos seien. Der neue Verband wollte die alten Tarifverträge nur mit spürbaren Kürzungen von Tarifnormen übernehmen: Das Urlaubsgeld sollte gestrichen, die 40-Stunden-Woche ohne Mehrarbeitszuschläge ermöglicht werden, eine vereinbarte Erhöhung der Jahressonderzahlung

29 Arens (1994): 1994 - Ein Ausblick, S. 3. 
um fünf Prozentpunkte zurückgenommen und die Konditionen für regelmäßige Samstagsarbeit verschlechtert werden..$^{30}$

In diesem Vorgehen sah die GTB eine neue Dimension der Versuche, Tarifbestimmungen zu schleifen, entschloss sich aber, nicht den vermutlich langwierigen juristischen Weg der Überprüfung zu gehen, sondern für den Abschluss der unveränderten Tarifverträge mit dem neuen Verband zu kämpfen. Mit der Plakatüberschrift "Der Wortbruch« und der Abbildung eines zerrissenen Tarifvertrages startete die Mobilisierung der Mitglieder in Bayern. Das Vorgehen der bayerischen Textilunternehmer erschreckte über die Branche hinaus und der DGB-Bundesvorstand unterstützte das Ringen gegen die juristische Trickserei der bayerischen Arbeitgeber materiell und personell. ${ }^{31}$

Für die Tarifgebiete der Textilindustrie mit Ausnahme Bayerns hatte Gesamttextil zentrale Verhandlungen gefordert, was die GTB ablehnte. Man verständigte sich jedoch darauf, den Tarifbezirk Westfalen/Osnabrück mit Pilotverhandlungen zu beauftragen. Diese Verhandlungen scheiterten, weil die Arbeitgeber einen Arbeitszeitkorridor durchsetzen und entweder Weihnachts- oder Urlaubsgeld kürzen wollten. Hätten sich die Arbeitgeber durchgesetzt, wäre die Reihe tarifwidriger betrieblicher Vereinbarungen für die Zukunft legalisiert worden. Trotz vieler Zugeständnisse auf betrieblicher Ebene verteidigte die Mehrheit der Tarifkommissionsmitglieder der starken Bezirke Westfalen/Osnabrück und Nordrhein die Regelungen zur Arbeitszeitflexibilisierung. Des Weiteren lehnten sie Kürzungen der Einmalzahlungen, wie sie in der Metall- und Elektroindustrie vereinbart worden waren, strikt ab. Die Textillöhne seien schließlich deutlich niedriger als bei Metall und auf diesem Gebiet sei eine Gleichbehandlung für die Textilarbeitgeber nie ein Thema gewesen. An diesen Gegensätzen scheiterte am 10. Juni 1994 auch der erneute Versuch, im Pilotbezirk Westfalen/Osnabrück zu einer Einigung zu kommen.

Zwei Wochen später verständigte sich die gewerkschaftliche Tarifkommission mit dem neugegründeten bayerischen Verband überraschend auf ein Tarifabkommen für das Tarifgebiet Nordbayern. Der breite Widerstand in den Betrieben und der Öffentlichkeit hatte zum Umdenken auf Arbeitgeberseite geführt; alle bisherigen Tarifverträge wurden unverändert vom neuen Verband übernommen. Wie beim tarifpolitischen Vorreiter, der Metall- und Elektroindustrie, stiegen die Löhne und Gehälter nach sechs Nullmonaten ohne Erhöhung um 2 Prozent. Der Ausgleichszeitraum für die regelmäßige wöchentliche Arbeitszeit konnte »in Ausnahmefällen « auf bis zu 52 Wochen ausgedehnt werden. Die bereits für 1994 und 1995 vereinbarten Erhöhungsstufen der Jahressonderzahlung um fünf Prozentpunkte wurden um jeweils ein Jahr verschoben - somit gab es keine Kürzung

30 GTB: Geschäftsbericht 1994-1997 des Hauptvorstandes, S. 266.

31 textil-bekleidung, Ausgabe 6/1994, S. 10. 
bereits zuvor gezahlter Tarifleistungen, sondern nur die Verschiebung der Verbesserung. ${ }^{32}$

Die GTB sprach zwar von einem "politischen Sieg «, doch bedeutete dieser Abschluss nicht den Durchbruch für alle Tarifgebiete, wie sich nur zehn Tage später im Tarifgebiet Südbayern zeigte. Da hier keine weitere Stufe bei der Erhöhung der Jahressonderzahlung vereinbart war, die verschoben werden konnte, forderten die Arbeitgeber die Kürzung der Jahressonderzahlung um fünf Prozentpunkte. Die Tariflandschaft war gespalten: In Tarifgebieten, in denen für die Zukunft Verbesserungen der Jahressonderzahlung vereinbart waren, wurden diese verschoben und damit die Tarifrunden beendet wie in Nordbayern. Für die Tarifgebiete Südbayern, Westfalen/Osnabrück und Nordrhein scheiterten die Verhandlungen an der Weigerung der gewerkschaftlichen Tarifkommissionen, geforderte Kürzungen der bestehenden Jahressonderzahlung zu akzeptieren.

Im Bezirk Westfalen/Osnabrück sah sich die Tarifkommission durch den Tarifabschluss in Nordbayern »verraten", weil man sich bundesweit darauf verständigt hatte, keine Eingriffe in bestehende Tarifverträge zuzulassen. Die Bayern wiesen jedoch auf ihre Sondersituation der aufgelösten Arbeitgeberverbände und die Notwendigkeit hin, mit dem neuen Verband die Tarifbindung wiederherzustellen. Sie interpretierten die Verschiebung der Erhöhungsstufen der Jahressonderzahlung so, dass nichts gekürzt werden könne, das noch nicht gezahlt worden ist.

Die Umsatzrückgänge und der Beschäftigungsabbau setzen sich 1994 unvermindert fort, die westdeutsche Textilindustrie strich nochmals über 10 Prozent der Arbeitsplätze. Die Bereitschaft der Beschäftigten zur Arbeitsniederlegung sank aufgrund der düsteren wirtschaftlichen Lage; selbst wenn Warnstreiks stattfanden, waren sie angesichts oft leerer Auftragsbücher kaum ein wirksames Druckmittel. Als Mitte September 1994 in einigen Tarifgebieten immer noch kein Tarifabschluss in Sicht war, bereitete die GTB im Tarifgebiet Westfalen/Osnabrück dennoch einen Arbeitskampf vor. Unmittelbar vor Beginn der geplanten Urabstimmungen über einen Streik wurde am 27. September 1994 ein Kompromiss erzielt: Nach acht Nullmonaten wurden die Löhne um 2 Prozent erhöht, der Ausgleichszeitraum für die regelmäßige tarifliche Wochenarbeitszeit wurde auf 52 Wochen verlängert. Die Jahressonderzahlung wurde nicht gekürzt, allerdings sollte die für Nordbayern und andere Bezirke festgelegte Verschiebung der 5-Prozent-Erhöhungsstufe bei der Tarifrunde 1995 im gleichen Wert berücksichtigt werden. Diese bedeutete die Kürzung der nächsten noch zu verhandelnden Tariferhöhung in Westfalen/Osnabrück um 0,4 Prozent.

Auf dieser Basis erfolgte schließlich auch der Abschluss in Südbayern mit Wiederinkraftsetzung aller bisherigen Tarifverträge. Dennoch zeigten sich wei- 
tere Schwierigkeiten. Die Tarifkommission Nordrhein verweigerte die Vorbelastung für die Tarifrunde 1995. In Baden-Württemberg dagegen befand man sich in einer besonderen Situation, hatte man doch 1990 keine außerordentliche Erhöhung der Jahressonderzahlung durchgesetzt, sondern sich stattdessen auf eine Nachschlags-Lohn- und Gehaltssteigerung geeinigt, so dass die Sonderzahlung hier deutlich niedriger ausfiel.

Es dauerte schließlich bis zum 12. Oktober 1994 und damit fünf Tage vor Beginn des 17. Ordentlichen Gewerkschaftstages, bis alle Tarifgebiete der Textilindustrie befriedet waren. In Nordrhein wurde die Vorbelastung für die Tarifrunde 1995 tatsächlich nicht vereinbart, dafür trat die Erhöhung der Löhne und Gehälter erst nach zehn Nullmonaten am 1. März 1995 in Kraft. In Baden-Württemberg wurde die Jahressonderzahlung sogar um 7,5 Prozentpunkte erhöht, dafür betrug die Lohnerhöhung ab 1. Januar 1995 aber nur 0,7 Prozent. Insgesamt hatte man sich dadurch in der Struktur der Tarifregelungen wieder den anderen Bezirken angenähert.

Von der Auflösung der bayerischen Arbeitgeberverbände war auch die Bekleidungsindustrie betroffen. Nach der bayerischen Einigung im Juni konnten die Bundesverhandlungen für die Bekleidungsindustrie nun weitergeführt werden. Am 18. Juli 1994 wurde ein Verhandlungsstand fixiert, der in den Bezirkstarifkommissionen beraten werden sollte. Die Lohn- und Gehaltserhöhung von 2 Prozent sollte ab 1. Dezember 1994, also nach sieben Nullmonaten wirksam werden. Die Arbeitszeitflexibilisierung würde innerhalb von 52 Wochen möglich sein, ohne dass wie bisher Zuschläge fällig würden. Die wöchentliche Höchstarbeitszeit wurde dabei auf 45 Stunden begrenzt.

Diese erweiterte Flexibilisierung entpuppte sich als Streitpunkt, denn nach den Tarifrichtlinien waren die Voten der Bezirkstarifkommissionen entscheidend. Gegen den erzielten Verhandlungsstand stimmten die Tarifkommissionen der großen Bekleidungsbereiche Bayern und Westfalen, alle anderen Bezirkskommissionen akzeptierten den Kompromiss. Der Manteltarifvertrag, der die Arbeitszeitgestaltung regelte, galt jedoch für das ganze Bundesgebiet. Am 3. Oktober 1994 entschied dann der zwanzigköpfige Hauptvorstand bei drei Gegenstimmen, den Kompromiss trotz des gegenteiligen Votums der Tarifkommissionen endgültig zu vereinbaren und damit die Tarifrunde auch für die Bekleidungsindustrie zu beenden.

\subsubsection{Aufarbeitung der Krisentarifrunden: Der Gewerkschaftstag 1994}

Die Tarifauseinandersetzung 1994 war die mit der höchsten Komplexität in der Geschichte der GTB und bestimmte die Diskussionen auf dem am 17. Oktober 1994 eröffneten Gewerkschaftstag. Elf Frauen und dreizehn Männer äußerten sich in der Aussprache zum Geschäftsbericht zur gerade abgeschlossenen Tarif- 
runde. Der für Tarifpolitik zuständige Winfried Hüren, im März 1994 vom Beirat für den zurückgetretenen Wolfgang Stender in den GHV gewählt, sah in der von einigen Arbeitgeberverbänden ermöglichten Mitgliedschaft ohne Tarifbindung die Aushöhlung der Tarifautonomie. ${ }^{33}$ Er kritisierte die Scheinheiligkeit der Verbände, die Tarifverträge unterzeichneten und im Anschluss deren Bruch verteidigten; diese Tarifbrüche würden dann wiederum als Waffe in Tarifauseinandersetzungen eingesetzt. Selbstkritisch fügte er mit Blick auf die Beteiligung vieler Betriebsräte hinzu:

»Wir können es uns nicht leisten, [...] wenn gewerkschaftliche Programmatik, unsere Reden und unsere Veröffentlichungen dem widersprechen, was sich in Betrieben tut. $\ll^{34}$

Die Reaktion der Delegierten war deutlich: In den Bekleidungsbetrieben Westfalens sei man »enttäuscht und auch etwas verbittert $«{ }^{35}$ Die Delegierten unterstrichen: »Wir sind vom Hauptvorstand falsch gebremst worden. $\aleph^{36}$ - Unsere Kolleginnen und Kollegen haben keinerlei Verständnis für diese Verschlechterungen. Vollzogene und angedrohte Austritte aus unserer Organisation sind die Folge. $\aleph^{37}-» \mathrm{Zu}$ hohe Belastungen für unsere Kolleginnen und Kollegen bei voller Ausschöpfung der tariflichen Möglichkeiten - bei Mehrbelastung werden gleichzeitig Einkommensverluste in Kauf genommen - das kann beides nicht Ziel gewerkschaftlicher Tarifpolitik sein. $\aleph^{38}$ - »Diese Tarifrunde war für uns im Betrieb schon ein Schlag ins Gesicht. ${ }^{39}$ Kritisiert wurde neben den Inhalten die unzureichende inhaltliche Beteiligung der Tarifkommissionen und das Überstimmen der kampffähigen Bereiche durch diejenigen mit schwächeren Strukturen.

Einzig die Delegierte Trude Kamp aus dem Tarifbezirk Nordrhein verteidigte die Tarifabschlüsse. Sie unterstrich die schwierigen wirtschaftlichen Rahmenbe-

33 Hüren (1994): Auch eine Frage der Ehre, S. 10.

34 GTB: Protokoll des 20. Ordentlichen Gewerkschaftstages, 17.-21. Oktober 1994 in Braunschweig, S. 73.

35 So die Delegierte Marita Mohning, in: GTB: Protokoll des 20. Ordentlichen Gewerkschaftstages, 17.-21. Oktober 1994 in Braunschweig, S. 91.

36 So der Delegierte Bernd Link, in: CTB: Protokoll des 20. Ordentlichen Gewerkschaftstages, 17.21. Oktober 1994 in Braunschweig, S. 93.

37 So die Delegierte Edith Echterdiek, in: GTB: Protokoll des 20. Ordentlichen Cewerkschaftstages, 17.-21. Oktober 1994 in Braunschweig, S. 95.

38 So der Delegierte Gerd Will, in: GTB: Protokoll des 20. Ordentlichen Gewerkschaftstages, 17.21. Oktober 1994 in Braunschweig, S. 97.

39 So der Delegierte Willi Mentemeier, in: GTB: Protokoll des 20. Ordentlichen Gewerkschaftstages, 17.-21. Oktober 1994 in Braunschweig, S. 109. 
dingungen und verwies auf die Tatsache, dass die Tarifverträge nur den Rahmen setzten, aber die Betriebsräte die konkreten Vereinbarungen träfen:

»Wenn ich an die kämpferischen Wortmeldungen hier denke, muss ich feststellen, dass die Betriebsrätinnen und Betriebsräte durchaus in der Lage sind, unberechtigte Forderungen der Arbeitgeber zurückzuweisen. ${ }^{40}$

Damit legte sie den Finger in die Wunde. Viele Funktionär:innen standen betrieblich unter Druck, erwarteten aber von ihrer Organisation, tarifvertraglich zu heilen, was betrieblich aufgegeben wurde.

Neben der Tarifpolitik für die Bekleidungsindustrie sahen die Delegierten insbesondere die Strategie in der Textilindustrie-Tarifrunde 1994 kritisch. Zwar sei es ein akzeptabler Weg gewesen, erstmals einen Pilotbezirk zu bestimmen. Man habe in Westfalen den Durchbruch versucht und den Kopf hingehalten, sei dann aber an der Kompromissfindung in Bayern nicht mehr beteiligt gewesen. Rolf Tschorn, Geschäftsführer der großen durch die Textilindustrie geprägten Verwaltungsstelle Emsdetten, betonte:

»Man darf jemandem, dem man als Vorreiter in einer Tarifrunde die Chance und das Vertrauen gibt, in die Tarifrunde hineinzugehen, nicht auf der Ziellinie die Zügel wegnehmen. $\aleph^{41}$

Auch in seiner Verwaltungsstelle habe der Tarifabschluss viele Mitgliederverluste zur Folge gehabt. Obwohl 35 Delegierte aus den neuen Bundesländern kamen, spielten der Zusammenbruch der dortigen Industrie und die Tarifpolitik für die verbliebenen 20.000 Beschäftigten in den Diskussionen auf dem Gewerkschaftstag keine Rolle. Die kritische Betrachtung der Tarifpolitik hatte Auswirkungen auf die Wahl des Führungsgremiums. Der Vorsitzende Willi Arens erhielt von den insgesamt 200 Delegierten nur 121 Stimmen (60,5 Prozent). Erst nach einer Bedenkzeit nahm er am folgenden Morgen die Wahl an. Die anderen Vorstandsmitglieder wurden dann mit mäßigen Ergebnissen in ihren Ämtern bestätigt. Der Gewerkschaftstag sprach sich gegen die sogenannte OT-Mitgliedschaft, d.h. die Mitgliedschaft von Unternehmen »ohne Tarifbindung« in Arbeitgeberverbänden aus. Flächentarifverträge

40 GTB: Protokoll des 20. Ordentlichen Gewerkschaftstages, 17.-21. Oktober 1994 in Braunschweig, S. 119.

41 GTB: Protokoll des 20. Ordentlichen Cewerkschaftstages, 17.-21. Oktober 1994 in Braunschweig, S. 102. 
»haben sich bewährt, indem sie auch der Konkurrenz zwischen Unternehmen gesellschaftlich vertragliche Grenzen setzen und den Arbeitnehmerinnen und Arbeitnehmern eine Mindestsicherung vor zwischenbetrieblicher Billigkonkurrenz sichern ${ }^{4}{ }^{42}$

Die GTB strebte weiter die 35-Stunden-Woche an, forderte das Recht auf Teilzeitarbeit, verbunden mit einem Rückkehranspruch auf einen Vollzeitarbeitsplatz und lehnte eine flexible Arbeitszeit ab, die zu unzumutbaren Belastungen führt. ${ }^{43}$ Weiter wurden das wichtige Ziel gemeinsamer Entgelttarifverträge für Arbeiter:innen und Angestellte beschlossen sowie gefordert, verstärkt die Interessen der Frauen in die Tarifpolitik einzubringen. Relevante Handlungsfelder wie Frauenförderung, familienfreundlichere Gestaltung der Arbeitszeit und die Höhergruppierung von typischer Frauenarbeit seien in die Tarifkonzepte aufzunehmen. Auf Antrag Baden-Württembergs beschloss der Gewerkschaftstag, die regionale Tarifpolitik für die Textilindustrie beizubehalten und dem Anliegen der Arbeitgeber, die die Verhandlungen lieber zentral führen wollten, entgegenzutreten.

\subsubsection{Schwierige Tarifrunde trotz Aufschwung}

1994 erholte sich die deutsche Wirtschaft. Nach dem Rezessionsjahr 1993 mit einer Schrumpfung der Wirtschaftsleistung um über I Prozent wuchs das deutsche Bruttoinlandsprodukt im Folgejahr wieder um 2,4 Prozent. ${ }^{44}$ Damit waren die gesamtwirtschaftlichen Vorzeichen für die Tarifrunden des Jahres 1995 positiv. Dem allgemeinen Aufschwung zum Trotz blieb die Inlandsproduktion der Bekleidungsindustrie mit minus 12,9 Prozent gegenüber dem Vorjahr weiter stark rückläufig, auch bei Textil verminderte sich die Produktion um 4,1 Prozent. ${ }^{45}$ Davon unbeeindruckt zeigten sich die GTB-Tarifkommissionen. So forderte die Gewerkschaft auf Grundlage der gesamtwirtschaftlichen Entwicklung Einkommenserhöhungen von 6 Prozent, um die Beschäftigten nicht von der allgemeinen Lohnentwicklung abzukoppeln.

Verbesserungen in gleicher Höhe hatte auch die IG Metall $\mathrm{zu}$ Beginn des Jahres 1995 gefordert. Doch nach Aufstellung der Forderungen machten die Metall-Arbeitgeber vier Monate lang kein Angebot für Tariferhöhungen, sondern

42 Entschließung 1: Tarifautonomie sichern, in: GTB: Protokoll des 17. Ordentlichen Gewerkschaftstages, 17.-21. Oktober 1994 in Braunschweig, S. 312.

43 Antrag 2 zur Tarifpolitik, in: GTB: Protokoll des 17. Ordentlichen Gewerkschaftstages, 17.-21. Oktober 1994 in Braunschweig, S. 313.

44 Statistisches Bundesamt (Destatis) (2018): Volkswirtschaftliche Gesamtrechnungen. Inlandsproduktberechnungen ab 1970, S. 14.

45 GTB: Geschäftsbericht 1994-1997 des Hauptvorstandes, S. 102. 
stellten wie in der Tarifrunde zuvor Gegenforderungen für Kostenentlastungen. Die IG Metall erklärte daraufhin die Verhandlungen für gescheitert und wählte statt des kampferprobten Baden-Württemberg das Tarifgebiet Bayern für den Arbeitskampf aus. Die bayerische Metallindustrie war mittelständisch geprägt, eine Großzahl von Betrieben stand am Ende der Lieferketten. Damit drohte keine Beeinträchtigung der Produktion in anderen Betrieben, denn für indirekt vom Streik betroffene Beschäftigte aus der Metall- und Elektroindustrie war nach der Änderung des \$116 Arbeitsförderungsgesetz die Zahlung von Kurzarbeitergeld ausgeschlossen. Die sogenannte Minimax-Strategie, mit möglichst wenig Streikenden eine möglichst große Wirkung durch die Fernwirkung zu erzielen, musste deshalb aufgegeben werden.

Nach elf bayerischen Streiktagen mit 34.000 Beteiligten erreichte die IG Metall eine Pauschalzahlung von 610 DM für vier Monate, daran anschließend eine Einkommenserhöhung von 3,4 Prozent für das laufende und 3,6 Prozent für das Folgejahr. ${ }^{46}$ Diese Tarifrunde wurde auf Arbeitgeberseite als Katastrophe eingeschätzt, wie Gesamtmetall in seiner 125-Jahre-Jubiläumsschrift im Jahr 2015 schrieb. ${ }^{47}$ Insbesondere Taktik und Verhandlungsführung waren innerhalb der Arbeitgebervereinigung heftig umstritten, waren die Arbeitgeber doch mit der Forderung nach einer kostenneutralen Null-Erhöhungsrunde in die Verhandlungen gegangen. Natürlich konnten sie diese Forderung nicht durchsetzen, aber selbst eine Erhöhung nur im Rahmen des Inflationsausgleichs konnte nicht erreicht werden. Hauptgeschäftsführer Dieter Kirchner musste nach 27 Jahren an der Spitze den Verband verlassen, Präsident Hans-Joachim Gottschol kandidierte nicht erneut. Gesamtmetall stand vor der Zerreißprobe.

Auch wenn das Scheitern der Totalverweigerungsstrategie von Gesamtmetall offensichtlich war, vermittelten die Textilarbeitgeber nicht den Eindruck, dass sie daraus Lehren gezogen hätten. Sie boten in den regionalen Tarifverhandlungen Einkommensverbesserungen von i Prozent an, vorausgesetzt, die GTB würde einem Arbeitszeitkorridor und Öffnungsklauseln für betriebliche Abweichungen zustimmen. Auch 25.000 Warnstreikende und die Einschaltung der Spitzenebene beider Seiten in die Nordrhein-Verhandlungen führten zu keinem Ergebnis, so dass für Nordrhein und Baden-Württemberg erneut die Vorbereitungen zu einem Arbeitskampf anliefen. Wie schon oft zuvor bot die Arbeitgeberseite nach dem Beschluss, Urabstimmungen durchzuführen, die Wiederaufnahme von Verhandlungen an. Für die GTB kamen Verhandlungen aber erst nach Ende der Urabstimmungen infrage, in denen sich 95,7 Prozent für einen Streik aussprachen. Mit diesem Druck konnte am 2. Juni 1995 auch bei den Textiler:innen die für die Metallindustrie vereinbarte Erhöhung der Tarifsätze durchgesetzt werden. Die

46 Gesamtmetall (2015): Tarifarchiv, Tabellen 6.1-6.11.

47 Gesamtmetall (2015): Der lange Weg zur Tarifpartnerschaft, S. 193-226 (auch zum Folgenden). 
Entgelte stiegen um 3,4 Prozent und für den ersten Monat gab es eine Pauschalzahlung von $100 \mathrm{DM}$.

Die GTB verpflichtete sich, über eine flexible Jahresarbeitszeit zu verhandeln. Sie veränderte ihre Strategie, sich in den Tarifrunden Stück für Stück mehr Flexibilität abpressen zu lassen, und nahm sich vor, ein eigenes Konzept zur Arbeitszeit $\mathrm{zu}$ entwickeln. Flexibilisierung sollte nicht nur den Betrieben nutzen, auch Beschäftigte sollten Möglichkeiten zu variabler Arbeitszeitgestaltung aus eigenem Interesse bekommen. ${ }^{48}$ Die veränderte gewerkschaftliche Position führte in der Schwesterbranche dazu, dass die festgefahrenen Tarifverhandlungen für die Bekleidungsindustrie am 6. Juni mit der Zusage abgeschlossen werden konnten, die Arbeitszeitgestaltung grundsätzlich neu zu diskutieren. Das Tarifergebnis brachte auch für die Bekleidungsbeschäftigten eine 3,4-prozentige Tariferhöhung ohne Nullmonate.

Trotz der deutlich schlechteren branchenwirtschaftlichen Situation hatte die GTB die gleiche Steigerung bei den Lohn- und Gehaltstarifverträgen durchgesetzt wie in der Metall- und Elektroindustrie. Die dazu nötige Streikvorbereitung bedeutete für die Organisation eine erhebliche organisatorische wie finanzielle Belastung, da sie aufgrund des deutlichen Rückgangs bei den Beitragseinnahmen Personal eingespart hatte. Hinzu kam, dass ein Streik die Kasse nochmals in großem Umfang belasten würde und die Erfolgsaussichten angesichts des vorherrschenden Auftragsmangels in vielen Betrieben unsicher waren.

Schon bei den Warnstreiks hatte eine Reihe von Arbeitgebern mit befristeten Aussperrungen reagiert - eine Reaktion, die in diesem Ausmaß nur von Textilunternehmern praktiziert wurde. So organisierten die Betriebe längere Stillstandzeiten, um die Einkommensverluste für die Beschäftigten zusätzlich zu erhöhen. Dennoch scheuten die Arbeitgeber das Risiko eines Arbeitskampfes, der einzelne Betriebe hart treffen konnte. Auf Solidarität im Unternehmerlager setzten sie nicht, sondern befürchteten im Gegenteil nicht ohne Grund, dass nicht bestreikte Konkurrenten gerne für zeitweise lieferunfähige Unternehmen einspringen würden.

\subsubsection{Der große Handschlag: Das Branchenbündnis Textil-Bekleidung}

Trotz des wirtschaftlichen Aufschwungs 1994 nahm die Arbeitslosigkeit in Deutschland weiter zu. Zum Jahresende hatte sie die 10-Prozent-Marke überschritten und stabilisierte sich auf diesem hohen Niveau. 1995 betrug sie in Ostdeutschland 14,8 und in Westdeutschland 9,1 Prozent. ${ }^{49}$ Vor diesem Hintergrund forderte der IG Metall-Vorsitzende Klaus Zwickel im Oktober 1995 ein gesell-

48 GTB: Geschäftsbericht 1994-1997 des Hauptvorstandes, S. 271.

49 Bundesagentur für Arbeit (2021): Tabellen-Arbeitslosigkeit im Zeitverlauf. 
schaftliches Bündnis für Arbeit. Durch Beiträge von Unternehmen, Staat und Gewerkschaften sollte eine Trendwende bei der Beschäftigung erreicht werden. Die Lohnforderungen könnten sich für einen gewissen Zeitraum nur an der Preissteigerung orientieren, die gesamtwirtschaftliche Produktivitätsentwicklung würde bei Tariferhöhungen unberücksichtigt bleiben.

Die Voraussetzungen dafür waren: Arbeitgeber und Regierung sollten im Gegenzug in den nächsten drei Jahren jährlich 100.000 neue Arbeitsplätze schaffen, 10.000 Langzeitarbeitslose einstellen und den Sozialabbau beenden. Der DGB nahm die Anregung auf und unterbreitete einen ähnlichen Vorschlag im Namen aller Mitgliedsgewerkschaften und für die gesamte deutsche Wirtschaft. Bundeskanzler Helmut Kohl ging zunächst auf die gewerkschaftlichen Angebote ein und man traf verbindliche Absprachen über Verhandlungen zu einem »Bündnis für Arbeit und Standortsicherung«. In sogenannten Kanzlerrunden sollte ein entsprechendes Maßnahmenpaket erarbeitet werden. ${ }^{50}$

Die Gespräche gerieten jedoch im ersten Quartal 1996 ins Stocken. Am 20. März 1996, schon vor dem offiziellen Scheitern, erklärte Gesamtmetall-Chef Werner Stumpfe das von der IG Metall vorgeschlagene Bündnis für Arbeit für "tot.$^{51}$ Als die Bundesregierung nach der aus ihrer Sicht erfolgreichen Landtagswahl in Baden-Württemberg die Gewerkschaften mit einem Sparpaket provozierte, war das Bündnis für die Gewerkschaften auf der gesamtwirtschaftlichen Ebene ebenfalls gescheitert. Insbesondere kam der Bruch zustande, weil die schwarz-gelbe Koalition die Lohnfortzahlung im Krankheitsfall auf 80 Prozent senken wollte. Zudem sollte der Kündigungsschutz in Betrieben mit bis zu zehn Beschäftigten ganz aufgehoben und soziale Leistungen für Arbeitslose und Familien mit Kindern eingeschränkt werden..$^{52}$

In der Textil- und der Bekleidungsindustrie setzte sich die wirtschaftliche Talfahrt ungebremst fort. 1995 gingen über 10 Prozent der Arbeitsplätze verloren, mehr als 30.000 Stellen wurden abgebaut. Nachdem die Tarifrunden für die Bekleidungsindustrie bereits 1994 und 1995 durch Spitzengespräche geschlichtet wurden, hatte sich zwischen den Akteuren ein persönliches Vertrauensverhältnis entwickelt. Ende 1995 suchten die Präsidenten und Geschäftsführungen der zentralen Arbeitgeberverbände den Kontakt zum GTB-Vorsitzenden. Die Verbandsspitzen argumentierten, in ihrem Lager sei es zunehmend schwierig, Unternehmer zu finden, die sich bereiterklärten, die vielen regionalen Verhandlungen zu führen. Außerdem gefährde der Stil der Auseinandersetzung die Mitgliedschaft in den Arbeitgeberverbänden.

50 DGB (o. J.): Der Weg zum ersten »Bündnis für Arbeit«.

51 Westdeutsche Zeitung, Ausgabe vom 21.3.1996.

52 DGB (o. J.): Der Weg zum ersten »Bündnis für Arbeit«. 
Die Arbeitgeber erklärten, sie seien zu einem Neuanfang bereit, und schlugen vor, künftig ausschließlich auf höchster Ebene und zudem für die Textil- und die Bekleidungsindustrie gemeinsam zu verhandeln. Dies sei ein entscheidender Beitrag, um das Tarifsystem zu stabilisieren, und zugleich die Voraussetzung zur Sicherung der noch bestehenden Arbeitsplätze, was auch dem von Zwickel vorgeschlagenen Bündnis entspreche.

Die Gremien der GTB berieten Anfang 1996 über diesen Arbeitgebervorschlag. Zwar gab es in den Tarifkommissionen eine grundsätzliche Skepsis gegen die Verlagerung der Tarifpolitik auf die Spitzenebene, jedoch war gerade in diesen Kommissionen angesichts des jahrelangen Arbeitsplatzverlustes und der fortwährenden Anstrengungen zur Durchsetzung der Reallohnsicherung der Kampfesmut geschwunden. Zum Zeitpunkt der Entscheidung über die Tarifforderungen 1996 gab es noch die sogenannten Kanzlerrunden zum Bündnis für Arbeit bei Helmut Kohl. Angesichts dieser Ausgangslage gab der GTB-Beirat, der die Tarifkonzeptionen zu genehmigen hatte, grünes Licht für Gespräche über ein umfassenderes Paket zu Tarifen und Beschäftigungssicherung. Im Beschluss dazu heißt es:

»Rund ein Drittel vernichteter Arbeitsplätze bei Textil und Bekleidung in den letzten drei Jahren allein in Westdeutschland erfordern auch von der CTB ein entschlossenes Handeln innerhalb eines Bündnisses, abgestellt auf die von uns betreuten Branchen. Um im Beschäftigungsrückgang der Textil- und Bekleidungsindustrie eine Wende einzuleiten und durch bessere Qualifizierung gleichzeitig auch die Zukunftschancen von Arbeitnehmerinnen und Arbeitnehmern dieser Industrie zu verbessern, strebt die GTB ein Textil-Bekleidungsbündnis für Beschäftigung und Ausbildung an. $\ll^{53}$

Bereits in den Monaten zuvor hatte die GTB-Tarifabteilung ein Konzept zur Arbeitszeitgestaltung erarbeitet, das mit allen Bezirkstarifkommissionen diskutiert wurde. Die Strategie, nun statt der bisher grundsätzlich abwehrenden Haltung offensiv mit einem eigenen Konzept in die Verhandlungen zu gehen, fand durchweg Zustimmung. Den Kern sollte ein verstetigtes Monatseinkommen auch für Arbeiter:innen bilden, die bisher nur nach geleisteten Stunden entlohnt wurden. Außerdem sollten die Flexibilisierungsmöglichkeiten der Betriebe durch den Anspruch der Beschäftigten auf Arbeitszeitautonomie ergänzt werden und auch die tarifvertragliche Gestaltung der Stufenausbildungen sowie Regelungen zur Fort- und Weiterbildung sollten in das Paket mit einbezogen werden.

Am selben Tag, als Gesamtmetall-Chef Werner Stumpfe das Bündnis für Arbeit für tot erklärte, verkündeten die Textil-Bekleidungs-Arbeitgeberverbände und die GTB ihre Einigung auf ein »Bündnis für Beschäftigung und Ausbildung«.

53 GTB Geschäftsbericht 1994-1997 des Hauptvorstandes, S. 276. 
Der kurze Verhandlungszeitraum, Form und Inhalt waren eine Zäsur der bisherigen Tarifpolitik. Gesamttextil und BBI bekannten sich in dieser »Bündnisvereinbarung " gemeinsam mit der GTB zum Flächentarifvertrag und forderten dazu auf, Verbände und Gewerkschaft zu stärken. Die Lohn- und Gehaltserhöhungen wurden für ein Jahr auf die Inflationsrate beschränkt, die zu diesem Zeitpunkt bei 1,5 Prozent lag.

Grundsätzlich wurde eine Jahresarbeitszeit auf der Basis der bisherigen 37-Stunden-Woche definiert, die bei gleichmäßigen Monatseinkommen betrieblich gestaltet werden konnte. Hinzu kam das Recht der Beschäftigten, bei Arbeitszeitguthaben autonom einzelne freie Tage in Anspruch zu nehmen. Zur Sicherung der Beschäftigung wurde auf der Basis freiwilliger - also weder durch den Betriebsrat noch den Arbeitgeber zu erzwingende ${ }^{54}$ - Betriebsvereinbarungen die Möglichkeit geschaffen, die Jahresarbeitszeit um bis zu 130 Stunden $\mathrm{zu}$ verkürzen oder zu erhöhen, und zwar mit entsprechender Kürzung oder Erhöhung der Entgelte. Solche Betriebsvereinbarungen waren nur bei Ausschluss betriebsbedingter Kündigungen für die Laufzeit dieser Vereinbarungen zulässig. Tarifliche Zuschläge konnten ab jetzt auch in Arbeitszeitguthaben umgewandelt werden.

Die überraschendste Neuerung war jedoch, dass die tarifliche Erhöhung der Löhne und Gehälter durch Vereinbarung mit dem Betriebsrat ganz oder teilweise verschoben werden konnte. Allerdings musste der vereinbarte Tariflohn bis zum Ende der Tariflaufzeit wieder erreicht sein. Damit wurden einkommenswirksame Teile des Tarifvertrags in die Disposition von Arbeitgebern und Betriebsräten gestellt. Die Möglichkeit, die Tariferhöhung zu verschieben, war an eine Beschäftigungszusage gekoppelt. Gleichzeitig sah der Tarifvertrag vor, dass die Lohnerhöhung bei guter wirtschaftlicher Lage durch Betriebsvereinbarung verdoppelt werden kann. Die Gespräche zu Aus- und Weiterbildungsfragen sollten weitergeführt werden. Grundsätzlich einigte man sich darauf, die Arbeit in den Industrien durch entsprechende Regelungen attraktiver zu machen. ${ }^{55}$

Diese Vereinbarung fand große öffentliche Resonanz, wie sie noch kein Tarifabschluss dieser Branche erfahren hatte. Das Kanzleramt ließ »Anerkennung« verlauten, Bundesarbeitsminister Blüm sandte Glückwünsche und Wirtschaftsminister Rexrodt sah das Bündnis für Arbeit durch die Textil- und Bekleidungstarifpartner wiederbelebt. Das Handelsblatt sprach vom Pilotabschluss für die gesamte Industrie, die Frankfurter Allgemeine Zeitung lobte die betrieblichen Freiheiten, die Rheinische Post betonte die Zukunftsausrichtung, die Westdeutsche Zeitung unterstrich die Ausrichtung auf die Beschäftigung und das Bran-

54 Freiwillige Betriebsvereinbarungen sind auch nicht durch Einigungsstellen erzwingbar, erfordern also immer die Zustimmung von Arbeitgeber und Betriebsrat. 
chenmedium »Textilwirtschaft « nannte die Vereinbarung gar eine tarifpolitische Sensation. ${ }^{56}$

Innerhalb des DGB war insbesondere die Öffnungsklausel zur Verschiebungsmöglichkeit der Einkommenserhöhung umstritten. Auch in der GTB wurde »vereinzelt Kritik laut«, wie die Mitgliederzeitschrift berichtete. Tarifkommissionsmitglieder fühlten sich von dem schnellen Tarifabschluss überrollt, da sie in die Spitzengespräche nicht eingebunden waren. Winfried Hüren, im GHV für Tarifpolitik zuständig, betonte den Einzelfallcharakter dieser Vereinbarung und sprach von einer absoluten Ausnahmesituation, die auf keinen Fall eine Kehrtwende in der Tarifpolitik darstelle. Er erwarte von den Arbeitgebern den Stopp des Arbeitsplatzabbaus und die Rückführung der gegen Tarifverträge verstoßenden betrieblichen Regelungen. ${ }^{57}$ Tatsächlich konnten durch diesen »Bündnis-Abschluss« die bislang gegen Tarifverträge verstoßenden betrieblichen Vereinbarungen eingefangen werden. Die Flächentarifverträge wurden gestärkt, in Westdeutschland blieb die Flucht aus Tarifverträgen der Textil- und Bekleidungsindustrie, sei es durch Mitgliedschaft ohne Tarifbindung oder Verbandsaustritt, die Ausnahme.

Im weiteren Verlauf des Jahres 1996 prägte das Handeln der Bundesregierung die Stimmung zwischen den Tarifvertragsparteien. Mit ihrem Sparpaket hatte die Kohl-Regierung die gesamtwirtschaftlichen Bündnisgespräche endgültig zum Scheitern gebracht. Die Gewerkschaften mobilisierten und Hunderttausende demonstrierten gegen den Sozialabbau. Trotz dieser Proteste beschloss der Bundestag am 13. September 1996, die Entgeltfortzahlung im Krankheitsfall von 100 auf 80 Prozent zu kürzen; die Regelung trat bereits zum 1. Oktober 1996 in Kraft. Diese Gesetzesänderung traf die Gewerkschaften ins Herz. 1957 hatte die IG Metall für die Lohnfortzahlung bei Krankheit den längsten Arbeitskampf ihrer Geschichte geführt. Die erste Große Koalition hatte die Arbeiter:innen 1969 den Angestellten gleichgestellt und so die ungekürzte Lohnfortzahlung im Krankheitsfall für alle Beschäftigten zum Gesetz werden lassen. ${ }^{58}$ Entgeltfortzahlungsregelungen waren in viele Manteltarifverträge aufgenommen worden.

Nach der Gesetzesänderung 1996 hatten sich die großen Arbeitgeberverbände darauf verständigt, alle tariflichen Vereinbarungen als rein deklaratorisch zu bezeichnen. Sie seien als reine Verweise auf die Gesetzeslage zu verstehen, deshalb gälten die dort enthaltenen Entgeltfortzahlungsregelungen nach der Gesetzesänderung nicht mehr. Die Metall-Arbeitgeberverbände forderten die Betriebe auf, das neue Entgeltfortzahlungsgesetz sofort umzusetzen und als erstes gro-

56 Zit. nach: textil-bekleidung, Ausgabe 4/1996, S. 7 (Presseschau) und Gesamttextil (1996): Bündnis für Beschäftigung und Ausbildung.

57 textil-bekleidung, Ausgabe 5/1996, S. 6 (»Das Bündnis steht - was sind die nächsten Schritte «).

58 Bundesgesetzblatt 1969, Teil I, S. 946. 
ßes Unternehmen erklärte Daimler-Benz öffentlich, die Lohnfortzahlungen zu kürzen. Die Beschäftigten »beim Daimler« protestierten umgehend und in den folgenden Tagen weiteten sich Arbeitsniederlegungen in der Metall- und Elektroindustrie aus; insbesondere in der Automobilindustrie legten die Beschäftigten mehrfach die Arbeit nieder. Die Metall-Arbeitgeberverbände kündigten die Tarifverträge über zusätzliches Urlaubsgeld und Sonderzahlungen, aber nicht die Bestimmungen über Entgeltfortzahlung im Krankheitsfall. ${ }^{59}$

Trotz des durch den »Bündnis-Abschluss« grundsätzlich entspannten Verhältnisses der Tarifparteien in der Textil- und der Bekleidungsindustrie forderten die Verbände auch hier die Betriebe auf, das Gesetz anzuwenden. Keine DGB-Gewerkschaft wollte die Frage, welche rechtliche Wirkung ihre Tarifverträge hätten, über den Rechtsweg klären, denn bis zu einer Entscheidung des Bundesarbeitsgerichtes würden Jahre vergehen. Stattdessen suchten die Gewerkschaften eine Lösung mit den Arbeitgeberverbänden.

Am 10. Oktober 1996 nahmen IG Metall und Gesamtmetall Verhandlungen über ein Gesamtpaket auf Spitzenebene auf. Zunächst verständigte man sich darauf, dass der Arbeitgeberverband seinen Mitgliedern empfiehlt, die Lohnfortzahlung vorerst bei 100 Prozent zu belassen. Im Gegenzug sagte die IG Metall zu, die Proteste auszusetzen. Die Verhandlungen auf Spitzenebene scheiterten, auch die folgenden regionalen Verhandlungen in Baden-Württemberg und NordrheinWestfalen endeten ohne Ergebnis.

Am 5. Dezember 1996 wurde in Niedersachsen ein Abschluss erzielt, ohne dass die Spitzen von IG Metall und Gesamtmetall eingebunden waren, indem ein Paket unter Einbezug der nächsten Einkommensrunden geschnürt wurde. Nach einer Pauschalzahlung von 200 DM für drei Monate, stiegen die Entgelte um 1,5 Prozent, zwölf Monate später wurden sie um weitere 2,5 Prozent angehoben. Die Einkommenstarifverträge liefen bis zum 31. Dezember 1998, also für zwei Jahre. Die Entgeltfortzahlung im Krankheitsfall betrug wieder 100 Prozent, jedoch wurden bei der zukünftigen Berechnung Mehrarbeitsvergütungen und -zuschläge nicht mehr berücksichtigt. Als Kompensation für die nun nicht wirksam werdende Kostenentlastung der Arbeitgeber durch die gekürzte Lohnfortzahlung wurde die tarifliche Sonderzahlung um fünf Prozentpunkte gekürzt. ${ }^{60}$

Auch die GTB hatte sich an den Protesten gegen die Kürzung der Entgeltfortzahlung beteiligt. Sie unterstützte betriebliche Aktionen, außerordentliche Betriebsversammlungen, Mehrarbeitsverweigerung und kurzfristige Arbeitsniederlegungen. Aufgrund der schlechten wirtschaftlichen Lage der meisten Betriebe und der Sorge der Beschäftigten um ihren Arbeitsplatz gelang es nur in

59 Cesamtmetall (2015): 125 Jahre Gesamtmetall-Der lange Weg zur Tarifpartnerschaft, S. 280.

60 Gesamtmetall (2015): 125 Jahre Gesamtmetall - Der lange Weg zur Tarifpartnerschaft, S. 296. 
wenigen Betrieben, die Entgeltfortzahlung in vollem Umfang zu sichern. ${ }^{61}$ Die bezirklichen Tarifkommissionen standen vor der Frage, wie eine Lösung gefunden werden könne. Trotz kritischer Stimmen zur zentralen Vereinbarung aus dem Frühjahr 1996 sah sich keine regionale Tarifkommission der Textilindustrie in der Lage, die Entgeltfortzahlung durchzusetzen. Schließlich forderten alle bezirklichen Kommissionen den Hauptvorstand auf, eine zentrale Lösung anzustreben. Aufgrund der Entwicklung der Metallindustrie war eine Lösung für die Entgeltfortzahlung nur in Verbindung mit der Tarifrunde 1997 möglich. Die von den Bezirkstarifkommissionen gewünschten zentralen Verhandlungen sollten diesmal von einer 23-köpfigen Hintergrundkommission begleitet werden.

In Sondierungsgesprächen mit den Arbeitgebern wurde ausgelotet, ob gemeinsame Verhandlungen für Textil und Bekleidung auf Bundesebene sinnvoll seien. Zunächst prallten dabei die grundsätzlich gegenteiligen Positionen aufeinander. Die GTB vertrat die Auffassung, dass die Entgeltfortzahlung in den meisten Tarifverträgen eigenständig gesetztes Recht war; die Arbeitgeber sprachen von der in der Praxis völlig problemlosen Umsetzung der gesetzlichen Regelung. Der Krankenstand habe sich teilweise dramatisch verringert. Wenn die Entgeltfortzahlung grundsätzlich bei 100 Prozent bliebe, müsse es - anders als in der Metall- und Elektroindustrie - eine »individuelle Kompensation" geben. Demnach sollten die Sonderzahlungen für diejenigen gekürzt werden, die krank waren und die Entgeltfortzahlung in Anspruch genommen hätten. ${ }^{62}$

Trotz der im Vorfeld nicht auszuräumenden grundsätzlich gegenteiligen Standpunkte wurde vereinbart, im Januar in offizielle zentrale Verhandlungen einzutreten. Schon nach zweitägigen Beratungen wurde am 17. Januar 1997 ein Ergebnis erzielt. Dabei wurde keine »normale« Gesamtvereinbarung unterschrieben, sondern die Fortsetzung des Bündnisses für Beschäftigung und Ausbildung vereinbart. Besonderheiten wie betriebliche Abweichungsmöglichkeiten oder der Freiraum für Arbeitszeitkorridore wurden fortgeschrieben. Die Entgeltfortzahlung von 100 Prozent im Krankheitsfall wurde ab März 1997 vereinbart, allerdings konnte die GTB nicht durchsetzen, dass kranke Arbeitnehmer:innen dadurch keine individuellen Nachteile hatten.

Den Betroffenen wurde weiterhin der volle Entgeltausfall bezahlt, aber für Krankheitstage wurde die Jahressonderzahlung um jeweils 1,5 Stundenentgelte gekürzt. Diese Regelung war für den ersten Krankheitsfall im Kalenderjahr auf vier Tage, für die folgenden drei Krankheitsfälle auf bis zu drei Tagen begrenzt. Da diese Regelung noch deutlich besser als die gesetzliche war, erfolgte eine weitere kollektive Kompensation der zusätzlichen Kosten für die Arbeitgeber durch die Kürzung der Jahressonderzahlungen um drei Prozentpunkte bei Textil und

61 GTB: Geschäftsbericht 1994-1997 des Hauptvorstandes, S. 278.

62 GTB: Geschäftsbericht 1994-1997 des Hauptvorstandes, S. 279. 
um 2,5 Prozentpunkte bei Bekleidung. Die Monatsentgelte wurden für 1997 um 1,5 Prozent und für 1998 um 2,1 Prozent erhöht. ${ }^{63}$

Neben den Regelungen zur Entgeltfortzahlung und der Erhöhung der Einkommen brachte die zweite »Bündnisvereinbarung« einen qualitativ neuen tarifpolitischen Schritt. Mit der Aufnahme der Förderung von Aus-, Fort- und Weiterbildung in den Tarifvertrag wurde erstmals ein Recht auf Bildungsfreistellung geschaffen. Die Arbeitgeber verpflichteten sich, bis zu 2 Prozent der Beschäftigten für eine Woche pro Jahr unter Fortzahlung ihrer Bezüge für Aus-, Fort- oder Weiterbildungsmaßnahmen freizustellen. Dazu wurde eine gemeinsame Bildungseinrichtung der Tarifvertragsparteien vereinbart, die durch Bildungsbeiträge aller Betriebe finanziert werden sollte. Aus diesem Fonds sollten die Kosten für Seminare, Reise, Unterkunft und Verpflegung finanziert oder zumindest bezuschusst werden. Pro Beschäftigtem wurden zunächst 7,50 DM, ab dem Folgejahr 10 DM abgeführt. Zum Ausgleich wurde die Erhöhung des Urlaubsgeldes um den entsprechenden Betrag gekürzt. Damit finanzierten die Beschäftigten indirekt den Fonds, die Arbeitgeber übernahmen den Entgeltausfall. ${ }^{64}$

Mehr als fünfzig Jahre zuvor hatte die GTB erstmals eine Bildungsfreistellung gefordert, jetzt war es ihr gelungen, diese tarifvertraglich zu verankern. Bemerkenswert ist, dass sich diese Regelung, die inzwischen finanziell weiter ausgebaut wurde, nicht nur auf Inhalte bezieht, die einen Bezug zur aktuellen beruflichen Tätigkeit haben. Das Vorschlagsrecht für die Hälfte der Teilnehmer:innen an Bildungsmaßnahmen und für die Verwendung der Fondsmittel hatte die GTB, heute liegt es bei der IG Metall. Hieraus ergibt sich ein eindeutiger Vorteil für Gewerkschaftsmitglieder.

Mit dem letzten Tarifabschluss der Gewerkschaft Textil-Bekleidung am 26. Februar 1997 konnte die Sicherung der Entgeltfortzahlung bei Krankheit, verbunden mit einer Entgelterhöhung von 2 Prozent für 1997 und Verbesserungen beim Urlaubsgeld, auch auf die neuen Bundesländer übertragen werden. Dem Tarifvertrag zur Aus-, Fort- und Weiterbildung verweigerten die ostdeutschen Unternehmer jedoch ihre Zustimmung.

\subsection{Wir können es schaffen - oder auch nicht}

Wie andere auch wurde die GTB im Herbst 1989 von der Dynamik der Entwicklungen in der DDR und Osteuropa überrascht. Mit der Öffnung der innerdeutschen Grenze am 9. November 1989 hatte das ostdeutsche SED-Regime endgültig die Autorität verloren. Die westdeutschen Gewerkschaften traf dieser Prozess unvor-

63 GTB: Geschäftsbericht 1994-1997 des Hauptvorstandes, S. 279.

64 GTB: Geschäftsbericht 1994-1997 des Hauptvorstandes, S. $279 f f$. 
bereitet; in den ersten Wochen nach Wende und Maueröffnung konnte niemand den weiteren politischen Verlauf voraussehen. Der GTB-Vorsitzende Berthold Keller warnte noch in der Januarausgabe 1990 der Mitgliederzeitschrift davor, angesichts der jüngsten Geschichte »nur unsere ureigenen Wünsche zum alleinigen Maßstab der politischen Entwicklung in Mitteleuropa [zu] machen « - trotz des »verständlichen Wunsches nach einem friedlichen Zusammenleben der Menschen in beiden Teilen Deutschlands ${ }^{65}{ }^{65} \mathrm{Als}$ wichtigste Entscheidungen des Jahres wurden die Wahlen in vier Bundesländern und die Bundestagswahl genannt.

Doch bereits im Dezember 1989 wurde deutlich, dass der Vorschlag von Bundeskanzler Helmut Kohl, über eine Annäherung zu einer Konföderation beider deutschen Staaten zu kommen, rasch überholt war. Es gab eine zunehmende Dynamik in Richtung staatliche Einheit. Mit der deutschen Wiedervereinigung war auch die Aufgabe der Schaffung einer Textil-Bekleidungs-Gewerkschaft für ganz Deutschland verbunden. Dieser Prozess vollzog sich ähnlich wie die staatliche Einheit nach dem Muster der alten Bundesrepublik, d.h. die GTB erweiterte ihre Zuständigkeit nun auf die neuen Bundesländer.

\subsubsection{Von den Träumen 1990 zur Wirklichkeit 1995}

Nach der DDR-Volkskammerwahl im März 1990 war der Weg zur staatlichen Einheit Deutschlands vorgezeichnet. Zwischen der westdeutschen GTB und der ostdeutschen IG Textil-Bekleidung-Leder (IG TeBeLe) gab es bereits seit Mitte der 1970er Jahre Kontakte, die im Rahmen der bundesdeutschen Entspannungspolitik begonnen und sich in den 1980er Jahren intensiviert hatten; im Frühjahr 1989 begann sogar ein gegenseitiger Jugendaustausch. Aufgrund der relativ guten Beziehungen zwischen GTB und IG TeBeLe eröffnete die GTB bereits im Januar 1990 ein Verbindungsbüro in Ostberlin. Die IG TeBeLe sollte auf dem Weg zu einer demokratisch aufgebauten Gewerkschaft begleitet werden. Als Unterorganisation des Freien Deutschen Gewerkschaftsbundes (FDGB) war sie nur beschränkt selbstständig und hatte keine eigene Finanzhoheit. Zur Wende waren in Ostdeutschland rund 300.000 Beschäftigte im Textil- und im Bekleidungssektor tätig. ${ }^{66}$

Wie andere Gewerkschaften und Organisationen in der DDR übte auch die Spitze der ostdeutschen Textilgewerkschaft im Frühjahr 1990 Selbstkritik, um die Kontrolle möglichst zu behalten. Man verselbstständigte zunächst die Organisation, um sich vom besonders diskreditierten FDGB zu distanzieren. Während dessen "Erneuerung « geradezu chaotisch verlief, behielten in der IG TeBeLe zumindest die Funktionär:innen der zweiten Ebene die Fäden in der Hand. Die Delegierten des ersten Kongresses der IG TeBeLe, die in den Betrieben frei gewählt

65 Keller (1990): Auf uns wartet noch viel Arbeit, S. 6.

66 Friedrich-Ebert-Stiftung (1992): Textilstandort Ostdeutschland, S. 3. 
worden waren, wählten nicht - wie vom Vorstand vorgesehen - den bisherigen Dresdener Bezirksleiter Hans-Joachim Breuer zum Vorsitzenden, sondern HansJürgen Nestmann. Der Geschäftsstellenleiter aus Aue war Funktionär der IG TeBeLe und zuvor auch SED-Mitglied gewesen; Breuer wurde zu seinem Stellvertreter gewählt. Allerdings war bei dieser Zentralkonferenz am 4. und 5. Mai 1990 schon klar, dass es künftig eine gemeinsame Gewerkschaft für ganz Deutschland geben würde, ${ }^{67}$ und bereits Ende September 1990 folgte der Beschluss, die IG TeBeLe zum Jahresende aufzulösen. ${ }^{68}$

Nach der deutschen Einheit waren mit einer Gewerkschaft Textil-Bekleidung für das wiedervereinigte Deutschland große Hoffnungen verbunden. Im November 1990 betonte der neue GTB-Vorsitzende Willi Arens: „Die Gewerkschaftseinheit wird unsere Gewerkschaft Textil-Bekleidung stärken. « $^{69}$ Trotz dieser positiven Einschätzung war die GTB beim Aufbau neuer Strukturen vorsichtig und etablierte in den Jahren 1991 und 1992 zunächst nur vorläufige Organisationsstrukturen, die trotzdem einen erheblichen Aufwand bedeuteten. Anstelle der satzungsgemäßen "Verwaltungsstellen« und »Bezirksleitungen« (siehe Kapitel 2.2.2) wurden zwanzig »Betreuungsstellen« und zwei »Koordinierungsstellen« eingerichtet. ${ }^{70}$

Anders als die IG Metall setzte die GTB in ihren 1991 eingerichteten ostdeutschen Büros ausschließlich Kolleg:innen ein, die schon in der DDR hauptamtlich für die Gewerkschaft gearbeitet hatten. Dies galt auch für die zwei übergeordneten »Koordinierungsstellen«, die von Hans-Jürgen Nestmann und Rosemarie Keller geleitet wurden. 1992 wurde Rosemarie Keller als Leiterin des Bezirkes Süd-Ost gewählt und war damit die einzige Bezirksleiterin der GTB. Trotz »Osterweiterung" blieb der GHV eine reine "Westveranstaltung", nur der ehrenamtliche Teil des Hauptvorstandes wurde neben den bisherigen Mitgliedern um zwei Kolleginnen und drei Kollegen aus den neuen Bundesländern erweitert. ${ }^{71}$ Die bisherigen 26 politischen Sekretär:innen in der Hauptvorstandsverwaltung wurden durch nur eine Kollegin aus dem neuen Organisationsgebiet verstärkt. ${ }^{72}$

Trotz der seit fast zwanzig Jahren sinkenden Mitgliederzahlen war die westdeutsche GTB finanziell gesund und hatte immer wieder bewiesen, dass sie auch unter schwierigen Rahmenbedingungen durchsetzungsfähig war. Nach der

67 textil-bekleidung, Ausgabe 6/1990, S. 14 f. (»Zentralkonferenz der IC Textil-Bekleidung-Leder. Blick nach vorn«).

68 textil-bekleidung, Ausgabe 10/1990, S. 3.

69 GTB: Protokoll des 16. Ordentlichen Cewerkschaftstages, 4.-9. November 1990 in Würzburg, S. 176.

70 GTB: Ceschäftsbericht 1990-1993 des Hauptvorstandes, S. 262.

71 CTB: Ceschäftsbericht 1990-1993 des Hauptvorstandes, S. 82.

72 CTB: Ceschäftsbericht 1990-1993 des Hauptvorstandes, S. 385. 
deutschen Wiedervereinigung sollte die Organisation mittelfristig weiter stabilisiert werden. Dazu stellte Serv Hennes, im GHV für Personal zuständig, im November 1990 einen Plan mit 112 zusätzlichen hauptamtlichen Stellen in Ostdeutschland vor. ${ }^{73}$ Doch aufgrund des raschen Zusammenbruchs der ostdeutschen Textil- und Bekleidungsindustrie und des anhaltenden Arbeitsplatzrückgangs in Westdeutschland fiel die zwischenzeitlich um über 100.000 gestiegene gesamtdeutsche Mitgliederzahl schon 1993 wieder auf den Stand von Ende 1990, also das Niveau der alten Bundesrepublik zurück. Der Traum einer Mitgliederstärkung wurde zum Albtraum der Finanznot. Zunächst ermöglichten die soliden Rücklagen die weitere Handlungsfähigkeit, doch der Rückgang der Mitgliederzahlen war nicht aufzuhalten.

Die Beitragseinnahmen von 55,9 Millionen DM 1990 in Westdeutschland stiegen gesamtdeutsch auf 69,2 Millionen DM im Jahr 1991, sanken aber im Folgejahr um 4,5 Millionen DM und im nächsten Jahr um weitere 7,2 Millionen DM. 1994 betrugen sie nur noch 52,4 Millionen DM, was Mindereinnahmen von knapp 25 Prozent innerhalb von nur drei Jahren bedeutete. ${ }^{74}$ Bereits im Jahr 1992 reichten die Beitragseinnahmen nicht mehr aus, um die nötigen Ausgaben zu decken; bis 1994 konnte die Finanzierungslücke durch Zinseinnahmen gedeckt werden. Der Spagat wurde notwendig, einerseits die Vertretung der Mitgliederinteressen weiter zu gewährleisten, andererseits aber die Ausgaben den Einnahmen anzupassen.

Maßnahmen zur finanziellen Stabilisierung wurden schon früh eingeleitet. Bis 1993 wurden dreizehn örtliche Verwaltungsstellen aufgelöst, in Westfalen und später in Bayern jeweils zwei Bezirke vereinigt. 1990 wurde der GHV von sieben auf sechs und 1994 auf fünf Mitglieder verkleinert; bis 1996 wurde die Anzahl der Verwaltungsstellen nochmals von 62 auf 36 reduziert. Die Beschäftigtenzahl sank von 413 Anfang 1991 auf 287 Ende 1995, die der politischen Sekretär:innen von 200 auf 145. Aufgrund der Altersstruktur innerhalb der GTB gelang dieser Anpassungsprozess fast ohne Kündigungen. ${ }^{75}$ Doch all diese Maßnahmen genügten nicht, um Ausgaben und Einnahmen wieder ins Gleichgewicht zu bringen, vor allem weil die Zahl der Branchenbeschäftigten weiter rapide sank. Ab 1995 musste zur Deckung der Ausgaben auf das Vermögen zurückgegriffen werden. ${ }^{76}$

Zugleich war der innerorganisatorische Anpassungsprozess sehr arbeitsintensiv. Betreuungsbereiche mussten neu zugeschnitten werden; durch die $\mathrm{Zu}$ -

73 GTB: Protokoll des 16. Ordentlichen Gewerkschaftstages, 4.-9. November 1990 in Würzburg, S. 63.

74 GTB: Geschäftsbericht 1990-1993 des Hauptvorstandes, S. 191; GTB: Geschäftsbericht 1994-1997 des Hauptvorstandes, S. 133.

75 Eigene Berechnungen aufgrund der Geschäftsberichte 1990-1993 und 1994-1997 des GTBHauptvorstandes.

76 GTB: Geschäftsbericht 1995-1997 des Hauptvorstandes, S. 134. 
sammenlegungen von Verwaltungsstellen litten die Kontakte zwischen Gewerkschaftssekretär:innen und betrieblichen Funktionär:innen. Die Wege zu den Gewerkschaftsbüros wurden weiter und die größeren Entfernungen hatten längere Anfahrtszeiten zu den Betrieben zur Folge. Unter diesen Voraussetzungen war es nicht mehr wie in der Vergangenheit möglich, den Beschäftigtenrückgang durch Erhöhung des Organisationsgrads auszugleichen, im Gegenteil: Ab 1993 ging die Mitgliederzahl stärker zurück als die der Beschäftigten, der Organisationsgrad sank empfindlich.

\subsubsection{Kooperation statt Fusion? Die "fünf kleinen Tiger"}

Auch wenn sich die Strukturschwierigkeiten bei der GTB besonders krass zeigten, so war die Situation auch in anderen Gewerkschaften angespannt. Die Mitgliederzahl der im DGB zusammengeschlossenen Gewerkschaften erhöhte sich mit der Wiedervereinigung von 7,9 Millionen auf 11,8 Millionen, aber bereits 1995 hatten die DGB-Gewerkschaften wieder 2,5 Millionen Mitglieder verloren. ${ }^{77}$ Weil alle Organisationen viel in den Aufbau ihrer Infrastruktur in den neuen Bundesländern investiert hatten, ergaben sich nun insbesondere für die kleineren DGBGewerkschaften Probleme wegen der zunehmenden Arbeitslosigkeit und der schwindenden Beitragszahlungen.

Schon zu Beginn der 1990er Jahre hatte die GTB eine Vereinigung mit der deutlich kleineren Gewerkschaft Leder angestrebt. In der DDR wie in internationalen Organisationen gehörte die lederverarbeitende Industrie zum gemeinsamen Organisationsbereich mit Textil-Bekleidung. Die Führung der Gewerkschaft Leder zog es jedoch vor, 1997 mit der IG Chemie, Papier, Keramik und der IG Bergbau und Energie zur neuen IG Bergbau, Chemie, Energie (IG BCE) zu fusionieren. Neben dem Zusammenschluss zu großen Multi-Branchen-Gewerkschaften betonten andere Organisationen den Weg der Selbstständigkeit, der eine höhere Identifikation der Mitglieder mit ihrer Gewerkschaft bedeute.

Trotz der schwierigen Umstände setzte sich die GTB 1993 das Ziel, das Jahr 2000 eigenständig zu erreichen. Der Hauptvorstand war mit den Funktionär:innen einer Meinung, dass zwar grundlegende Veränderungen notwendig seien, die Organisationskultur einer über hundert Jahre gewachsenen Gewerkschaft aber erhalten bleiben müsse. Neben Maßnahmen zur Verschlankung und Steigerung der Effektivität erarbeitete die GTB gemeinsam mit der Gewerkschaft Nahrung, Genuss, Gaststätten (NGG), der Gewerkschaft Holz und Kunststoff (GHK) und der IG Medien das Modell einer verstärkten Kooperation als Alternative zur Fusion; später kam die Gewerkschaft Handel, Banken und Versicherungen (HBV)

77 Greef (2014): Gewerkschaften im Spiegel von Zahlen, Daten und Fakten, S. 688. 
hinzu. Auf dem Gewerkschaftstag 1994 beschrieb der GTB-Vorsitzende Willi Arens die vergleichbare Ausgangslage der Kooperationsgewerkschaften:

»Erstens. Mitgliederrückgang zwingt zum Rückzug aus der Fläche. Zweitens. Mittelbau und Zentrale müssen so ausgedünnt werden, dass Serviceaufgaben für die Untergliederungen nicht darunter leiden. ${ }^{78}$

Dies sollte durch Arbeitsteilung und organisationsübergreifende Betreuung erreicht werden. Als ersten Schritt öffneten die »fünf kleinen Tiger« ihre Büros als Anlaufstellen für alle Mitglieder der fünf Organisationen, um ihnen trotz der Schließung vieler örtlicher Verwaltungsstellen kurze Wege zum Gewerkschaftsbüro zu ermöglichen. Darüber hinaus sollte die Zusammenarbeit in weiteren Bereichen verstärkt werden, um finanzielle Ressourcen einzusparen.

Der Gewerkschaftstag 1994 betonte die Bereitschaft zur Infragestellung aller Strukturen und zur Vertiefung der Kooperation unter Einbezug des personellen Einsatzes und der Vereinfachung der Verwaltung. Vorrang habe die Eigenständigkeit der GTB. In Anträgen aus Südbayern und Baden-Württemberg wurde zur Voraussetzung gemacht, »daß von den Beitragseinnahmen entsprechende Rücklagen in den Streikfonds für Arbeitskämpfe gebildet werden« und »daß die Ausgaben den Beitragseinnahmen mittelfristig so angepasst sind, daß die gewerkschaftliche Arbeit [...] gesichert ist «. Sollte dies nicht gelingen, sei der Hauptvorstand aufgefordert, »rechtzeitig neue Vorschläge zu erarbeiten, die auch den Zusammenschluss mit anderen Gewerkschaften in Erwägung ziehen ${ }^{79}$

Das Konzept des Erhalts der Selbstständigkeit war an die Verschlankung der Organisation und die entlastend wirkende Kooperation mit den anderen vier Gewerkschaften gebunden. Die mit der NGG bereits 1978 vereinbarte Kooperation (siehe Kapitel 6.4) funktionierte dort gut, wo es konkrete Vereinbarungen zur Zusammenarbeit gab wie bei der Herausgabe einer gemeinsamen Mitgliederzeitung. Die ebenfalls gewünschte Zusammenarbeit auf anderen Ebenen hatte jedoch kaum Früchte getragen. Aufgrund dieser Erfahrung traten GTB und NGG in den Gesprächen mit den zusätzlichen Partnern für konkrete und verbindliche Absprachen ein, denn nur so könnten durch Arbeitsteilung echte Synergien entstehen. Die Gewerkschaften könnten politisch weiter eigenständig bleiben, müssten ihre Apparate jedoch operativ zusammenführen, und zwar sowohl in der Fläche als auch in den Vorstandsverwaltungen. Dies bedeutete, dass bislang eigenständig bearbeitete Aufgaben zur Disposition gestellt werden mussten.

78 GTB: Protokoll des 17. Ordentlichen Cewerkschaftstages, 17.-21. Oktober 1994 in Braunschweig, S. 50.

79 Beschlossener Antrag Org6, in: GTB: Protokoll des 17. Ordentlichen Cewerkschaftstages, 17.21. Oktober 1994 in Braunschweig, S. 334. 
Der im November 1994 unterzeichnete Kooperationsvertrag der fünf Partner benennt die möglichen Felder: Verwaltung der Mitgliederdateien, gemeinsame Publikationen für Mitglieder wie z. B. Materialien für Betriebsräte und Vertrauensleute, Rechtsschutz, Bildungsarbeit und gemeinsame Büros in den Regionen. Doch obwohl die GTB im Jahr 1995 erneut fast 12 Prozent ihrer berufstätigen Mitglieder verlor, kamen die Gespräche über die Konkretisierung der Zusammenarbeit der »fünf kleinen Tiger« nicht voran. GTB und NGG strebten nun die Zusammenarbeit der fünf Gewerkschaften im Sinne einer »Teilfusion« an: 50 bis 60 regionale Büros sollten gemeinsam genutzt, wesentliche Aufgaben der Vorstandsverwaltungen unter den Kooperationsgewerkschaften aufgeteilt und nur von einer Organisation bearbeitet werden. Doch so weit wollten die anderen drei Gewerkschaften nicht gehen. Sie verfolgten ein Konzept der eher losen Zusammenarbeit auf noch zu definierenden Feldern, wobei die Organisationen weitgehend unverändert bleiben sollten.

Da der GTB-Vorsitzende 1995 als Jahr der Entscheidung ausgerufen hatte, begann angesichts der bescheidenen Fortschritte in Sachen Entlastung durch Kooperation nun die Diskussion über ein mögliches Zusammengehen mit einer oder mehreren DGB-Gewerkschaften. ${ }^{80}$

\subsubsection{Aufgabe der Selbstständigkeit: IG Metall statt IG BCE}

Der Preis für die ausgedünnte Betreuungsstruktur war ein sinkender Organisationsgrad; die Talfahrt bei der Mitgliederentwicklung der GTB setzte sich fort. Darüber hinaus hielt die Verlagerung der Bekleidungsproduktion in Niedriglohnländer unvermindert an und auch die Produktion von Textilien für Bekleidung folgte dieser Entwicklung. Im Januar 1996 stellten die Kooperationsgewerkschaften offiziell fest, dass sie sich nicht auf den von GTB und NGG favorisierten Weg verständigen konnten. Daher lag es nahe, dass die beiden schon enger kooperierenden Gewerkschaften die Fusion zu einer Konsumgütergewerkschaft prüften. Doch die Gespräche der Geschäftsführenden Vorstände von NGG und GTB zeigten, dass die Einspareffekte nicht ausreichen würden, um eine langfristig handlungsfähige Organisation zu bilden. ${ }^{81}$

Nun musste sich die GTB also nach anderen, stärkeren Partnern umsehen. Mit der IG Chemie, Papier, Keramik gab es einen engen Berührungspunkt in der Chemiefaserherstellung, die zu deren Organisationsbereich gehörte. Außerdem hatte es schon unter dem Vorsitz von Berthold Keller eine enge politische Zusammenarbeit mit der IG Chemie gegeben und die Kontakte auf der Vorstandsebe-

80 Verein zur Weiterbildung der Arbeitnehmerinnen und Arbeitnehmer in der Textil- und Bekleidungsindustrie (1998): Die GTB 1990 bis 1998, S. $72 \mathrm{ff}$.

81 GTB: Ceschäftsbericht 1994-1997 des Hauptvorstandes, S. 74f. 
ne waren immer noch gut. In der Chemiegewerkschaft ging man davon aus, der Partner der Wahl für eine Fusion zu sein, sollte doch auch die Lederverarbeitung zur neu zu gründenden IG Bergbau, Chemie, Energie (IG BCE) gehören. Aber im April 1996 zeigte sich auch die IG Metall an einem Anschluss der GTB interessiert. Die politischen Gegensätze der 1980er Jahre waren verblasst, organisatorische Anknüpfungspunkte gab es bereits durch Automobilzulieferer-Unternehmen, die teilweise in den Verbänden der Textilindustrie organisiert waren. Hier hatte es in Einzelfällen bereits Streit um die gewerkschaftliche Zuständigkeit gegeben.

In den Gesprächen mit der IG Chemie und der IG Metall orientierte sich die GTB an der Kernfrage, wie die »textile Identität« bewahrt werden könne. Dabei ging es insbesondere um die Bereitschaft der aufnehmenden Gewerkschaft, auf allen Ebenen satzungsgemäße Strukturen zu schaffen, in denen sich auch die textilen Ehrenamtlichen wiederfinden. Die Tarifpolitik sollte in der neuen Organisation nicht über einen Kamm geschoren, sondern wie bisher mitgliedernah mit den Branchen-Tarifkommissionen weiterentwickelt werden. Weitere entscheidende Punkte waren der Erhalt der Werner-Bock-Schule in Beverungen und die Fortführung der Stiftung für die Beschäftigten der Miederindustrie, die die "Kritische Akademie« in Inzell betrieb (siehe Kapitel 4.2). Kriterien waren auch die angemessene Vertretung in den Beschlussorganen und die Übernahme der GTB-Beschäftigten. ${ }^{82}$

Sowohl die IG Metall als auch die IG Chemie erklärten sich bereit, durch Veränderungen ihrer Organisation die Voraussetzungen für eine wirksame organisatorische wie branchen- und tarifpolitische Vertretung zu schaffen und die textile Identität zu bewahren. Die Mitglieder des GHV tendierten in der Mehrheit zur IG Chemie. Zwar hatte die GTB in der Tarifpolitik stets gekämpft und war alles andere als ein »Schwarm von Friedfischen «, aber die oft wahrgenommene sprachliche Härte der IG Metall klang vor allem in den Ohren vieler Bekleidungsbeschäftigten fremd. Allerdings tendierten die GTB-Funktionär:innen aus der »zweiten Reihe« der Hauptvorstandsverwaltung bis zu den Verwaltungsstellen mehrheitlich zur IG Metall. Sie versprachen sich bessere persönliche Entfaltungsmöglichkeiten und auch wohnortnähere Einsätze aufgrund des dichteren Verwaltungsstellennetzes.

Hinzu kam als wichtige Komponente der Unterschied in Größe und Finanzkraft zwischen IG Metall und IG Chemie. Ohne dass dies offen diskutiert wurde, hatte die IG Chemie-Spitze in den Gesprächen klargemacht, dass sie höchstens zwei zusätzliche Büros in textilen Schwerpunktregionen einrichten werde, in denen sie bislang nicht mit eigenen Verwaltungsstellen vertreten war. Außerdem ging sie von einem Personalüberhang aus, der kurzfristig abzubauen wäre.

82 Verein zur Weiterbildung der Arbeitnehmerinnen und Arbeitnehmer in der Textil- und Bekleidungsindustrie (1998): Die GTB 1990-1998, S. 79. 
Die IG Chemie hatte bereits die Gewerkschaft Leder integriert und war mit den Vorbereitungen für den Zusammenschluss mit der IG Bergbau und Energie beschäftigt. Entgegenkommender war die IG Metall, die die Übernahme der GTBBeschäftigten nicht einschränkte. Hinzu kam, dass sie dreimal so viele Verwaltungsstellen vor Ort hatte wie die IG Chemie und den GTB-Mitgliedern wieder ortsnahe Anlaufstellen bieten konnte. ${ }^{83}$

Für die IG Metall war von Bedeutung, dass nicht alle fusionsbereiten Gewerkschaften von der IG Chemie aufgenommen werden, damit sich diese nicht als einzige Multibranchen-Industriegewerkschaft neben der IG Metall positionierte. Da das Vermögen der aufzunehmenden GTB deren Verpflichtungen - insbesondere die der betrieblichen Altersversorgung - überstieg, konnte man die laufenden Personalkosten verkraften. Die IG Metall sicherte auch zu, die Tarifpolitik auf der Grundlage der bestehenden Regelungen weiterzuführen, ohne dabei grundlegende Kurswechsel anzustreben. ${ }^{84}$

Am 27. Juni 1996 beschloss der GTB-Hauptvorstand, dem Beirat vorzuschlagen, auf einem außerordentlichen Gewerkschaftstag im Jahr 1997 die Voraussetzungen für eine Fusion mit der IG Metall zu schaffen. Dies sei aber keine Entscheidung gegen die in der Gründungsvorbereitung befindliche IG Bergbau, Chemie, Energie. Im Beschluss hieß es weiter, dass der Hauptvorstand

»in der innerorganisatorischen Diskussion und Willensbildung eine sich abzeichnende breite Mehrheit für eine Fusion mit der IC Metall [erkenne]. Die wesentlich größere Anzahl der Verwaltungsstellen ermöglicht bei unseren spezifischen Branchen-, Betriebs- und Mitgliederstrukturen eine effektive betriebsnahe Betreuung und realistische wohnortnahe Beteiligungsangebote, auch an die Mitglieder, die betrieblich nicht mehr zu erfassen sind. ${ }^{85}$

Damit war der überwiegenden Stimmung Rechnung getragen. Dennoch gab es unter den Funktionär:innen auch kritische Stimmen, denen die Mitgliederzeitung in der Septemberausgabe Gehör verschaffte. Zwei Betriebsratsvorsitzende aus der Bekleidungsindustrie befürchteten, dass ihre mittlerweile recht kleine Branche wenig Chancen habe, ihre Interessen in diesem Umfeld deutlich zu machen oder den richtigen Ton zu treffen. ${ }^{86}$ Die Mehrheit der von der Mitgliederzei-

83 Diese Darstellung beruht auf einem Interview der Verfasser:innen am 25. Mai 2020 mit Manfred Schallmeyer, bis 1998 Mitglied im Geschäftsführenden Hauptvorstand der GTB und anschließend geschäftsführendes Vorstandsmitglied der IC Metall.

84 Informationen aus dem Interview der Verfasser:innen mit Manfred Schallmeyer am 25. Mai 2020.

85 GTB: Geschäftsbericht 1994-1997 des Hauptvorstandes, S. 75.

86 textil-bekleidung, Ausgabe 9/1996, S. 10f. (»Zwischen Skepsis und großer Zustimmung«). 
tung befragten Funktionär:innen sah jedoch keine Alternative zur Auflösung der GTB und verband mit der Integration in die IG Metall eher Chancen als Probleme.

Die Integrationsverhandlungen fanden im Jahr der großen Auseinandersetzung mit der Regierung Kohl statt, die 1996 mit einem Sparpaket die Axt an die 100-prozentige Entgeltfortzahlung im Krankheitsfall und damit an eine hart erkämpfte Errungenschaft gelegt hatte. Parallel zu den anlaufenden Verhandlungen zur konkreten Ausgestaltung der Integration hatte die GTB in schwieriger Zeit ihr Tagesgeschäft weiter zu führen, außerdem musste sie ihren »BündnisTarifabschluss « innerorganisatorisch aufarbeiten (siehe Kapitel 7.1.6). Im ersten Halbjahr wurde der Integrationsvertrag zwischen GTB und IG Metall vereinbart. Man hatte sich darauf verständigt, nicht mehr von einer Fusion, sondern angesichts der doch sehr unterschiedlichen Größenverhältnisse von einer Integration der GTB in die IG Metall zu sprechen. Der GTB-Beirat billigte den Vertrag bei einer Gegenstimme.

Auf dem 3. Außerordentlichen Gewerkschaftstag der GTB vom 29. September bis 1. Oktober 1997 in Neuss begründete Manfred Schallmeyer, Mitglied des GHV, den Antrag auf Auflösung der Organisation:

»Unter den obwaltenden Umständen und Rahmenbedingungen, von denen der wirtschaftliche, organisatorische, finanzielle und personelle Teil des vorgelegten Ceschäftsberichtes handelt, überstieg es immer mehr unsere Kräfte, die Ansprüche zu erfüllen, die eine Gewerkschaft an sich selbst stellen muss, wenn sie bundesweit präsent, als Tarifvertragspartei nicht demütiger Bittsteller, sondern selbstbewusst den Ton angeben und als Organisation mitgliederstark und finanzkräftig sein will. Kurzum: Es überstieg unsere Kräfte, eine Gewerkschaft bleiben zu wollen, an der man einfach nicht vorbeikommen kann. ${ }^{87}$

Wehmut, aber auch Aufbruchsstimmung waren spürbar. Die Delegierte Heidi Wlecke erinnerte an die Pläne der letzten drei Jahre: Selbstständigkeit, Kooperation, Fusion mit der NGG, dann Gespräche mit der IG Chemie und der IG Metall: Wir auf der unteren Ebene, in den Vorständen, [...] in den Betrieben konnten teilweise gar nicht so schnell und so rasant umdenken. " $^{88}$ Günter Röttger sah die Schwierigkeit, »bei einer so großen Organisation ein Bein auf die Erde zu kriegen ${ }^{89}$ Auch Wolfgang Lehnhardt berichtete von viel Skepsis im Betrieb und in

87 CTB: Protokoll des 3. Außerordentlichen Gewerkschaftstages, 29. September-1. Oktober 1997 in Neuss, S. 81.

88 CTB: Protokoll des 3. Außerordentlichen Gewerkschaftstages, 29. September-1. Oktober 1997 in Neuss, S. 86.

89 CTB: Protokoll des 3. Außerordentlichen Gewerkschaftstages, 29. September-1. Oktober 1997 in Neuss, S. 90. 
der Verwaltungsstelle: »Es herrschen Ängste vor, Ängste in die Richtung, dass wir uns als Funktionäre der GTB in der großen IG Metall nicht mehr wiederfinden werden. ${ }^{90}$ Edith Echterdiek betonte die Eigenverantwortung in der neuen Organisation:»Kämpfen können wir, das haben wir in der Vergangenheit bewiesen. [...] Es liegt an uns, an unserer Stärke, ob wir im Aufwärtsgang mit hoch erhobenem Kopf oder im Rückwärtsgang in die IG Metall gehen. «I $^{91}$

Der Gewerkschaftstag beschloss einstimmig bei nur einer Enthaltung die Auflösung der Gewerkschaft Textil-Bekleidung zum 30. Juni 1998. In diesem Beschluss wurde betont, dass die IG Metall die Gewähr für die Kontinuität der Tarifarbeit, eine flächendeckende Präsenz von Verwaltungsstellen, Raum für Branchenarbeit und ein zielgerichtetes Bildungsangebot für die Gewerkschafts- und Betriebsratsarbeit in Klein- und Mittelbetrieben biete. ${ }^{92}$

\subsection{Vom Aufbruch bis zum Aufgeben: Die Ära Willi Arens}

Bei der Wahl von Willi Arens im Jahr 1990 waren die historischen politischen Lager innerhalb der GTB bereits bedeutungslos. Die Stimmergebnisse bei den Vorstandswahlen hingen vielmehr von rhetorischen Fähigkeiten, Geschick oder Ungeschick im Umgang mit wichtigen Funktionär:innen und der Positionierung in aktuellen Fragen ab. Willi Arens war mit hinreichendem Ergebnis zum Vorsitzenden gewählt worden (siehe Kapitel 7.1).

Das schwache Stimmergebnis 1990 für Wolfgang Stender, der 1989 vom Beirat für Hermann Schumacher nachgewählt worden und für Tarifpolitik zuständig war, kam jedoch fast einer Niederlage gleich. Stender hatte sich als rechte Hand von Berthold Keller bereits einige Gegner gemacht. In der Tarifpolitik sah sich der selbstbewusste Gewerkschafter mit nicht minder selbstbewussten Bezirksleitern konfrontiert. Im Geschäftsführenden Hauptvorstand (GHV) gab es die gewohnte Lagermentalität nicht mehr, durch die die Mitglieder der sogenannten »Familie«, der informellen Führungsriege der GTB, stets gestützt worden waren. Insbesondere die Bezirksleiter aus Westfalen, Baden-Württemberg und Nordrhein drängten schon für die Tarifrunde 1994 auf einen Wechsel. Dem konnte sich Stender nicht mehr widersetzen und trat Anfang 1994 »aus persönlichen und gesundheit-

90 CTB: Protokoll des 3. Außerordentlichen Gewerkschaftstages, 29. September-1. Oktober 1997 in Neuss, S. 91.

91 GTB: Protokoll des 3. Außerordentlichen Gewerkschaftstages, 29. September-1. Oktober 1997 in Neuss, S. 89.

92 Politische Erklärung und Auflösungsbeschluss, in: GTB: Protokoll des 3. Außerordentlichen Gewerkschaftstages, 29. September-1. Oktober 1997 in Neuss, S. $185 \mathrm{ff}$. 
lichen Gründen« zurück. Der Beirat wählte den nordrheinischen Bezirksleiter Winfried Hüren in den GHV nach.

Da die Verkleinerung des GHV für 1994 bereits beschlossen war, wurde die Position des im Dezember 1993 verstorbenen Kassierers Serv Hennes nicht mehr neu besetzt. Die Zuständigkeit für die Finanzen übernahm der Stellvertretende Vorsitzende Hermann Paschen.

Bei der Wiederwahl von Arens im Jahr 1994 gestalteten sich die Ereignisse dramatisch. Angesichts einer Zustimmung von nur 60,5 Prozent (121 Stimmen bei 200 Delegierten) sah sich der sichtbar enttäuschte Vorsitzende außerstande, umgehend über die Wahlannahme zu entscheiden und der Gewerkschaftstag wurde unterbrochen. Am nächsten Morgen appellierte der Stellvertretende Vorsitzende Hermann Paschen im Namen des Hauptvorstandes und der Bezirksleiter, »im Interesse der Organisation die Wahl anzunehmen«. Dies tat Willi Arens dann auch mit einer längeren Erklärung. Er betonte, mit Kritik gut leben zu können, kritisierte aber die mangelnde sachliche Auseinandersetzung mit ihm und seinen Entscheidungen und setzte sich so mit »einem Teil der hauptamtlichen Sekretäre« auseinander, der seinen Frust in geheimen Wahlen abreagiere. ${ }^{93}$

In der Tat gab es insbesondere im Bezirk Baden-Württemberg viele Hauptamtliche, denen die Amtsführung von Arens einschließlich seiner »westfälischen Sturheit« missfiel. Der baden-württembergische Bezirksleiter Werner Heindel hatte selbst mit einer Kandidatur geliebäugelt, diese aber aus gesundheitlichen Gründen verworfen. Auch dem GHV-Mitglied Manfred Schallmeyer wurden Ambitionen auf den Vorsitz nachgesagt. Hätte er Willi Arens herausgefordert, hätte er eine große Chance gehabt, die Mehrheit zu erzielen. Unmittelbar nach Bekanntgabe des Wahlergebnisses gehörte er jedoch zu denjenigen, die Willi Arens das Vertrauen aussprachen und ihn baten weiterzumachen.

Auch die anderen GHV-Mitglieder wurden wiedergewählt: Hermann Paschen als Stellvertretender Vorsitzender (165 Stimmen), Manfred Schallmeyer (164 Stimmen), Winfried Hüren (144 Stimmen) und Waltraud Hessedenz (125 Stimmen).

Wie Berthold Keller startete Willi Arens seine Amtszeit programmatisch, und zwar mit einer »Modernisierung« des 1978 verabschiedeten »Mannheimer Programms« der GTB. Dies sei keine modische Verbeugung vor dem Zeitgeist, vielmehr schrieb Arens im Vorfeld des Gewerkschaftstages 1990:

»[...] mit dem Programm machen wir deutlich, daß sich die GTB verändern will und muß. Verändern müssen sich angesichts des tiefgreifenden gesellschaftlichen Wandels manche Inhalte und Formen unserer gewerkschaftlichen Arbeit«. ${ }^{94}$

93 CTB: Protokoll des 17. Ordentlichen Cewerkschaftstages, 17.-21. Oktober 1994 in Braunschweig, S. 138.

94 Arens (1990): GTB-Programm, S. 3. 
Arens setzte sich für eine gleichberechtigte Aufnahme der Umweltpolitik neben der Wachstums- und Strukturpolitik in die GTB-Programmatik ein - ein Thema, das seine Organisation immer noch zu nachrangig behandle, wie er auf dem Gewerkschaftstag kritisch anmerkte. Arens sah die Welt stärker durch die »Zerstörung der natürlichen Lebensgrundlagen " gefährdet als durch äußere Feinde. ${ }^{95}$ Ebenso legte er Wert darauf, das Thema "Europäische Gemeinschaft" im überarbeiteten Programm zu stärken. Diese müsse zu einem gemeinsamen Haus weiterentwickelt werden. ${ }^{96}$ Angesichts der Aktualität und Dringlichkeit, die beide Themen heute kennzeichnen, war Arens seiner Zeit voraus. Zwar hatte die Frage der europäischen Zusammenarbeit schon einen gewissen Stellenwert im Gewerkschaftslager, den der neue GTB-Vorsitzende aber spürbar verstärkte. Bei der Umweltpolitik hingegen schieden sich bei den Gewerkschaften die Geister, weil manche darin Gefahren für den Industriestandort Deutschland sahen.

Außerdem unterstrich Arens den Aspekt der Gleichberechtigung von Frau und Mann. Hier sei die GTB mit ihrem Programm von 1978 der Diskussion in CDU und SPD weit voraus gewesen, allerdings müsse man intensiver an der Verwirklichung arbeiten - es reiche nicht aus, das Thema nur ins Programm zu schreiben. ${ }^{97}$ 1990 stand Willi Arens für eine neue Zeit in der GTB, die nicht mehr von den alten Kämpfen um die politische Ausrichtung bestimmt war. Er warb für neue Ideen und die kritische Betrachtung bestehender Strukturen und liebgewordener Rituale. Die Gewerkschaft wollte er für junge Menschen attraktiver machen: Unkonventionelle jugendliche Vorstellungen seien keine Bedrohung der Organisation, sondern eine Bereicherung, die den Fortschritt beflügeln könne. ${ }^{98}$

Die deutsche Einheit und der darauffolgende Zusammenbruch der Textil- und der Bekleidungsindustrie in den neuen Bundesländern, die Wirtschaftskrisen und die drohende Erosion der Flächentarifverträge auch in den alten Bundesländern machten eine neue Qualität von Gewerkschaftspolitik erforderlich. Umbau und Anpassung der Organisation wurden durch die rasch geringer werdenden Einnahmen mehr erzwungen als planvoll gestaltet. Diese externen Einflüsse schränkten den Gestaltungsspielraum des Vorsitzenden deutlich ein.

In den Jahren 1993 und 1994, die durch verschärfte Branchenkrisen geprägt waren, gelang es Arens, das Augenmerk der Politik auf die krisengebeutelte Tex-

95 GTB: Protokoll des 16. Ordentlichen Cewerkschaftstages, 4.-9. November 1990 in Würzburg, S. 180.

96 GTB: Protokoll des 16. Ordentlichen Cewerkschaftstages, 4.-9. November 1990 in Würzburg, S. 241.

97 GTB: Protokoll des 16. Ordentlichen Gewerkschaftstages, 4.-9. November 1990 in Würzburg, S. 242.

98 GTB: Protokoll des 16. Ordentlichen Cewerkschaftstages, 4.-9. November 1990 in Würzburg, S. 184 . 
til- und Bekleidungswirtschaft zu lenken. Wirksame Strukturhilfen für die unter Sozial- und zunehmend auch unter Ökodumping leidende Branche konnte er jedoch nicht durchsetzen. Das Bundeswirtschaftsministerium blieb auf liberalem Kurs, schließlich vollzog sich das Sterben der Textil- und der Bekleidungsbranche nur in kleinen regionalen Einheiten. Der Fokus der Politik lag in diesen Krisenzeiten auf größeren Branchen wie der Automobilindustrie. Noch desaströser zeigte sich die Lage in Ostdeutschland, wo es nicht einmal gelang, die hier ehemals bedeutende Textilindustrie wenigstens im Kern zu erhalten. Der Kampf um mittelfristige Lohnkostenzuschüsse zur Anpassung an die massive Verteuerung der Produkte, die mit Einführung der D-Mark erfolgt war, blieb erfolglos. Schließlich sahen die meisten westdeutschen Unternehmen ihr Heil in der Auslandsverlagerung der Produktion; die liberalen Wirtschaftsminister verweigerten jede Form von Strukturbeihilfen.

Willi Arens, tarifpolitisch zeitlebens ein Pragmatiker, zeigte zunehmend seine Abneigung gegen die gewachsenen Strukturen und Hakeleien im Klein-Klein der Tarifpolitik. Er war davon überzeugt, dass man mit den Verbandsspitzen zu vernünftigen Regelungen kommen könne, sofern beide Seiten ihre Scheu überwinden und auch einmal über den eigenen Schatten springen würden. Ab 1994 mischte er sich auch operativ in die Lösung der Tarifkonflikte ein, 1996 und 1997 nutzte er die schwierigen Rahmenbedingungen und den schwindenden Kampfgeist in den Tarifkommissionen, um die sogenannten »Bündnis-Tarifabschlüsse« auszuhandeln und damit die Struktur der Tarifpolitik grundsätzlich und nachhaltig zu verändern (siehe Kapitel 7.1.6).

Die Meinungen der mächtigen Bezirksleiter waren nicht die Richtschnur für Arens' Handeln. Er konnte durchaus mit Kritik leben, aber sobald er sich eine Meinung gebildet hatte, tat er sich mit anderen Argumenten schwer. Persönlich bescheiden, reagierte er schroff, wenn er bei den Hauptamtlichen Anspruchsdenken wahrzunehmen meinte. Anders als sein Vorgänger Berthold Keller war Willi Arens kein »Haudrauf « und auch niemand, der hinter jeder Meinungsäußerung wirklich oder vermeintlich Andersdenkender eine Provokation oder gar eine Falle sah. Im persönlichen Umgang war Arens unprätentiös, galt jedoch auch nicht als begnadeter Redner.

Noch auf dem Gewerkschaftstag im Herbst 1994 überwog die Hoffnung, die politische Eigenständigkeit durch die kostenentlastende Kooperation mit anderen Gewerkschaften erhalten zu können. Ein Jahr später war klar, dass das nicht funktionieren würde. Arens steuerte die GTB in Richtung Fusion, zunächst mit dem klaren Ziel, Teil der neuen IG Bergbau, Chemie, Energie zu werden. Er ließ sich jedoch von den Vorzügen der Integration in die IG Metall überzeugen, weil er die Meinung der Jüngeren ernst nahm. Auf dem Auflösungskongress der GTB betonte er die Aufgabe der Gewerkschaften, den Dienstleistungssektor besser zu erschließen. Er beschwor die Notwendigkeit, junge Menschen stärker in den Fokus 
$\mathrm{zu}$ nehmen und sich darüber Gedanken zu machen, wie man Auszubildende in Kleinbetrieben ansprechen und gewinnen könne. ${ }^{99}$ Die Neuordnung der Gewerkschaftslandschaft war aus Arens' Sicht nur sinnvoll, wenn die durch Synergieeffekte frei werdenden Ressourcen genutzt würden, um die Gewerkschaftsarbeit auf die veränderten Wirtschafts- und Beschäftigtenstrukturen einzustellen.

Mit der Integration der GTB in die IG Metall im Jahr 1998 ging Willi Arens in den Ruhestand. Er starb am 5. Januar 2011 im Alter von 73 Jahren an den Folgen einer Operation in Hannover.

99 CTB: Protokoll des 3. Außerordentlichen Gewerkschaftstages, 29. September - 1. Oktober 1997 in Neuss, S. 46. 
